\title{
SYZYGIES OF ORIENTED MATROIDS
}

ISABELLA NOVIK, ALEXANDER POSTNIKOV, and BERND STURMFELS

\begin{abstract}
We construct minimal cellular resolutions of squarefree monomial ideals arising from hyperplane arrangements, matroids, and oriented matroids. These are StanleyReisner ideals of complexes of independent sets and of triangulations of Lawrence matroid polytopes. Our resolution provides a cellular realization of $R$. Stanley's formula for their Betti numbers. For unimodular matroids our resolutions are related to hyperplane arrangements on tori, and we recover the resolutions constructed by D. Bayer, S. Popescu, and B. Sturmfels [3]. We resolve the combinatorial problems posed in [3] by computing Möbius invariants of graphic and cographic arrangements in terms of Hermite polynomials.
\end{abstract}

\section{Cellular resolutions from hyperplane arrangements}

A basic problem of combinatorial commutative algebra is to find the syzygies of a monomial ideal $M=\left\langle m_{1}, \ldots, m_{r}\right\rangle$ in the polynomial ring $\mathbf{k}[\mathbf{x}]=\mathbf{k}\left[x_{1}, \ldots, x_{n}\right]$ over a field $\mathbf{k}$. One approach involves constructing cellular resolutions, where the $i$ th syzygies of $M$ are indexed by the $i$-dimensional faces of a CW-complex on $r$ vertices. After reviewing the general construction of cellular resolutions from [4], we define the monomial ideals and resolutions studied in this paper.

Let $\Delta$ be a $C W$-complex (see $[12, \S 38]$ ) with $r$ vertices $v_{1}, \ldots, v_{r}$, which are labeled by the monomials $m_{1}, \ldots, m_{r}$. We write $c \geq c^{\prime}$ whenever a cell $c^{\prime}$ belongs to the closure of another cell $c$ of $\Delta$. This defines the face poset of $\Delta$. We label each cell $c$ of $\Delta$ with the monomial $m_{c}=\operatorname{lcm}\left\{m_{i} \mid v_{i} \leq c\right\}$, the least common multiple of the monomials labeling the vertices of $c$. Also, set $m_{\emptyset}=1$ for the empty cell of $\Delta$. Clearly, $m_{c^{\prime}}$ divides $m_{c}$ whenever $c^{\prime} \leq c$. The principal ideal $\left\langle m_{c}\right\rangle$ is identified with the free $\mathbb{N}^{n}$-graded $\mathbf{k}[\mathbf{x}]$-module of rank 1 with generator in degree $\operatorname{deg} m_{c}$. For a pair of cells $c \geq c^{\prime}$, let $p_{c}^{c^{\prime}}:\left\langle m_{c}\right\rangle \rightarrow\left\langle m_{c^{\prime}}\right\rangle$ be the inclusion map of ideals. It is a degree-preserving homomorphism of $\mathbb{N}^{n}$-graded modules.

DUKE MATHEMATICAL JOURNAL

Vol. 111, No. 2, (C) 2002

Received 16 June 2000. Revision received 6 February 2001.

2000 Mathematics Subject Classification. Primary 05B35, 13D02; Secondary 05E99, 13 F55.

Postnikov's work partially supported by National Science Foundation grant number DMS- 9840383.

Sturmfels's work partially supported by National Science Foundation grant number DMS-9970254. 
Fix an orientation of each cell in $\Delta$, and define the cellular complex $C_{\bullet}(\Delta, M)$,

$$
\cdots \stackrel{\partial_{3}}{\longrightarrow} C_{2} \stackrel{\partial_{2}}{\longrightarrow} C_{1} \stackrel{\partial_{1}}{\longrightarrow} C_{0} \stackrel{\partial_{0}}{\longrightarrow} C_{-1}=\mathbf{k}[\mathbf{x}],
$$

as follows. The $\mathbb{N}^{n}$-graded $\mathbf{k}[\mathbf{x}]$-module of $i$-chains is

$$
C_{i}=\bigoplus_{c: \operatorname{dim} c=i}\left\langle m_{c}\right\rangle,
$$

where the direct sum is over all $i$-dimensional cells $c$ of $\Delta$. The differential $\partial_{i}: C_{i} \rightarrow$ $C_{i-1}$ is defined on the component $\left\langle m_{c}\right\rangle$ as the weighted sum of the maps $p_{c}^{c^{\prime}}$ :

$$
\partial_{i}=\sum_{c^{\prime} \leq c, \operatorname{dim} c^{\prime}=i-1}\left[c: c^{\prime}\right] p_{c}^{c^{\prime}},
$$

where $\left[c: c^{\prime}\right] \in \mathbb{Z}$ is the incidence coefficient of oriented cells $c$ and $c^{\prime}$ in the usual topological sense. For a regular $\mathrm{CW}$-complex, the incidence coefficient $\left[c: c^{\prime}\right]$ is +1 or -1 , depending on the orientation of cell $c^{\prime}$ in the boundary of $c$. The differential $\partial_{i}$ preserves the $\mathbb{N}^{n}$-grading of $\mathbf{k}[\mathbf{x}]$-modules. Note that if $m_{1}=\cdots=m_{r}=1$, then $C_{\bullet}(\Delta, M)$ is the usual chain complex of $\Delta$ over $\mathbf{k}[\mathbf{x}]$. For any monomial $m \in \mathbf{k}[\mathbf{x}]$, we define $\Delta_{\leq m}$ to be the subcomplex of $\Delta$ consisting of all cells $c$ whose label $m_{c}$ divides $m$. We call any such $\Delta_{\leq m}$ an $M$-essential subcomplex of $\Delta$.

PROPOSITION 1.1 ([4, Prop. 1.2])

The cellular complex $C_{\bullet}(\Delta, M)$ is exact if and only if every $M$-essential subcomplex $\Delta_{\leq m}$ of $\Delta$ is acyclic over $\mathbf{k}$. Moreover, if $m_{c} \neq m_{c^{\prime}}$ for any $c>c^{\prime}$, then $C_{\bullet}(\Delta, M)$ gives a minimal free resolution of $M$.

Proposition 1.1 is derived from the observation that, for a monomial $m$, the (deg $m$ )graded component of $C_{\bullet}(\Delta, M)$ equals the chain complex of $\Delta_{\leq m}$ over $\mathbf{k}$. If both of the hypotheses in Proposition 1.1 are met, then we say that $\Delta$ is an $M$-complex, and we call $C_{\bullet}(\Delta, M)$ a minimal cellular resolution of $M$. Thus each $M$-complex $\Delta$ produces a minimal free resolution of the ideal $M$. In particular, for an $M$-complex $\Delta$, the number $f_{i}(\Delta)$ of $i$-dimensional cells of $\Delta$ is exactly the ith Betti number of $M$, that is, the rank of the $i$ th free module in a minimal free resolution. Thus, for fixed $M$, all $M$-complexes have the same $f$-vector.

Examples of $M$-complexes appearing in the literature include planar maps (see [11]), Scarf complexes (see [2]), and hull complexes (see [4]). A general construction of $M$-complexes using discrete Morse theory was proposed by E. Batzies and $\mathrm{V}$. Welker [1]. We next introduce a family of $M$-complexes which generalizes those in [3, Th. 4.4].

Let $\mathscr{A}=\left\{H_{1}, H_{2}, \ldots, H_{n}\right\}$ be an arrangement of $n$ affine hyperplanes in $\mathbb{R}^{d}$,

$$
H_{i}=\left\{v \in \mathbb{R}^{d} \mid h_{i}(v)=c_{i}\right\}, \quad i=1, \ldots, n,
$$


where $c_{1}, \ldots, c_{n} \in \mathbb{R}$ and $h_{1}, \ldots, h_{n}$ are nonzero linear forms that span $\left(\mathbb{R}^{d}\right)^{*}$.

We fix two sets of variables $x_{1}, \ldots, x_{n}$ and $y_{1}, \ldots, y_{n}$, and we associate with the arrangement $\mathscr{A}$ two functions $m_{x}$ and $m_{x y}$ from $\mathbb{R}^{d}$ to sets of monomials:

$$
m_{x}: v \longmapsto \prod_{i: h_{i}(v) \neq c_{i}} x_{i} \quad \text { and } \quad m_{x y}: v \longmapsto\left(\prod_{i: h_{i}(v)>c_{i}} x_{i}\right) \cdot\left(\prod_{j: v_{j}(v)<c_{j}} y_{j}\right) .
$$

Note that $m_{x}(v)$ is obtained from $m_{x y}(v)$ by specializing $y_{i}$ to $x_{i}$ for all $i$.

\section{Definition 1.2}

The matroid ideal of $\mathscr{A}$ is the ideal $M_{\mathscr{A}}$ of $\mathbf{k}[\mathbf{x}]=\mathbf{k}\left[x_{1}, \ldots, x_{n}\right]$ generated by the monomials $\left\{m_{x}(v): v \in \mathbb{R}^{d}\right\}$. The oriented matroid ideal of $\mathscr{A}$ is the ideal $O_{\mathscr{A}}$ of $\mathbf{k}[\mathbf{x}, \mathbf{y}]=\mathbf{k}\left[x_{1}, \ldots, x_{n}, y_{1}, \ldots, y_{n}\right]$ generated by $\left\{m_{x y}(v): v \in \mathbb{R}^{d}\right\}$.

The hyperplanes $H_{1}, \ldots, H_{n}$ partition $\mathbb{R}^{d}$ into relatively open convex polyhedra called the cells of $\mathscr{A}$. Two points $v, v^{\prime} \in \mathbb{R}^{d}$ lie in the same cell $c$ if and only if $m_{x y}(v)=m_{x y}\left(v^{\prime}\right)$. We write $m_{x y}(c)$ for that monomial and, similarly, $m_{x}(c)$ for its image under $y_{i} \mapsto x_{i}$. Note that $m_{x}\left(c^{\prime}\right)$ divides $m_{x}(c)$, and $m_{x y}\left(c^{\prime}\right)$ divides $m_{x y}(c)$, provided $c^{\prime} \leq c$. The cells of dimension zero and $d$ are called vertices and regions, respectively. A cell is bounded if it is bounded as a subset of $\mathbb{R}^{d}$. The set of all bounded cells forms a regular CW-complex $B_{\mathscr{A}}$ called the bounded complex of $\mathscr{A}$.

Figure 1 shows an example of a hyperplane arrangement $\mathscr{A}$ with $d=2$ and $n=4$, together with monomials that label its bounded cells. The bounded complex $B_{\mathscr{A}}$ of this arrangement consists of 4 vertices, 5 edges, and 2 regions.

\section{THEOREM 1.3}

(a) The ideal $M_{\mathscr{A}}$ is minimally generated by the monomials $m_{x}(v)$, where $v$ ranges over the vertices of $\mathscr{A}$. The bounded complex $B_{\mathscr{A}}$ is an $M_{\mathscr{A}}$-complex. Thus its cellular complex $C_{\bullet}\left(B_{\mathscr{A}}, M_{\mathscr{A}}\right)$ gives a minimal free resolution for $M_{\mathscr{A}}$.

(b) The ideal $O_{\mathscr{A}}$ is minimally generated by the monomials $m_{x y}(v)$, where $v$ ranges over the vertices of $\mathscr{A}$. The bounded complex $B_{\mathscr{A}}$ is an $O_{\mathscr{A}}$-complex. Thus its cellular complex $C_{\bullet}\left(B_{\mathscr{A}}, O_{\mathscr{A}}\right)$ gives a minimal free resolution for $O_{\mathscr{A}}$.

To prove Theorem 1.3, we must check that for both ideals the two hypotheses of Proposition 1.1 are satisfied. The second hypothesis is immediate: for a pair of cells $c>c^{\prime}$, there is a hyperplane $H_{i} \in \mathscr{A}$ that contains $c^{\prime}$ but does not contain $c$, in which case $m_{x}(c)$ is divisible by $x_{i}$ and $m_{x}\left(c^{\prime}\right)$ is not divisible by $x_{i}$. The same is true for the oriented matroid ideal $O_{\mathscr{A}}$. The essence of Theorem 1.3 is the acyclicity 


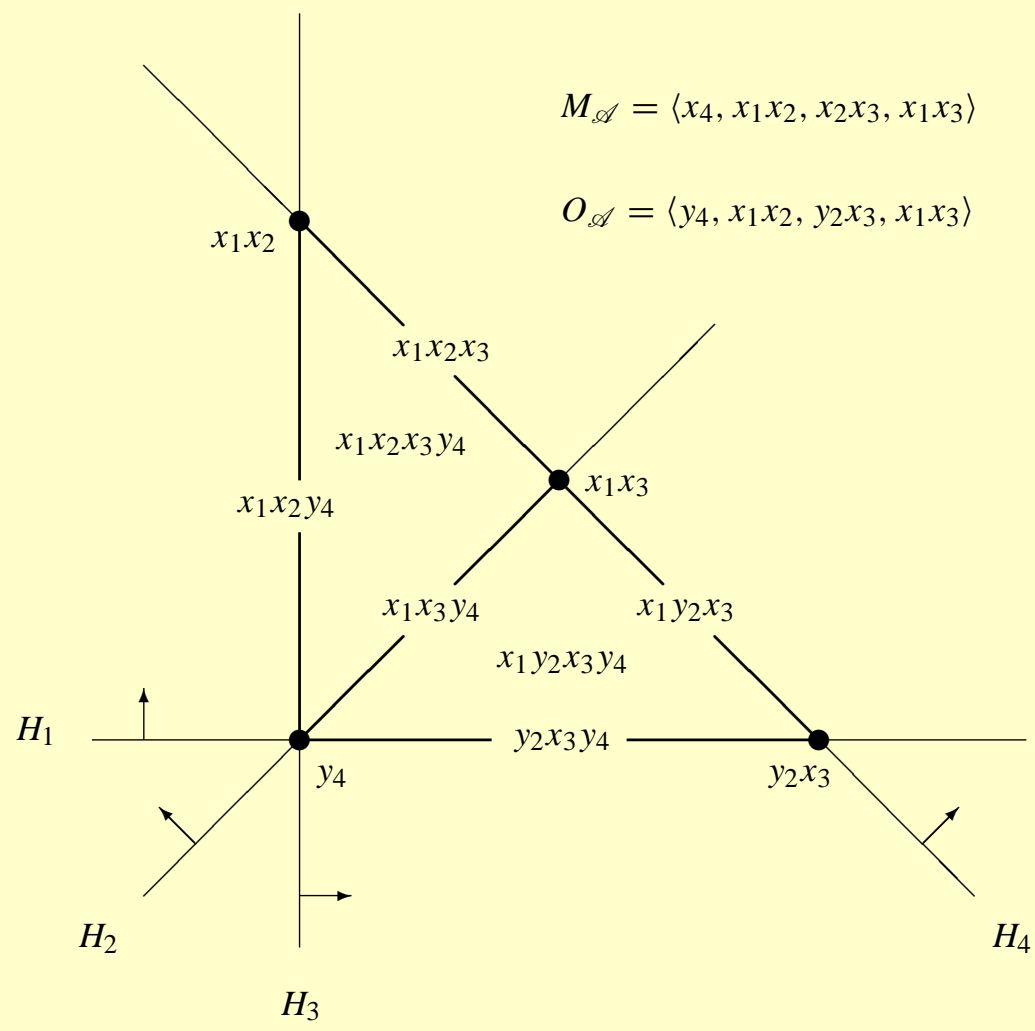

Figure 1. The bounded complex $B_{\mathscr{A}}$ with monomial labels

condition, which states that all $M_{\mathscr{A}}$-essential and $O_{\mathscr{A}}$-essential subcomplexes of $B_{\mathscr{A}}$ are acyclic. For the whole bounded complex, the following proposition is known.

PROPOSITION 1.4 (Björner and Ziegler (see [6, Th. 4.5.7]))

The complex $B_{\mathscr{A}}$ of bounded cells of a hyperplane arrangement $\mathscr{A}$ is contractible.

The acyclicity of all $M_{\mathscr{A}}$-essential subcomplexes of $B_{\mathscr{A}}$ is an easy consequence of Proposition 1.4: each $M_{\mathscr{A}}$-essential subcomplex is a bounded complex of a hyperplane arrangement induced by $\mathscr{A}$ in one of the flats of $\mathscr{A}$. The acyclicity of all $O_{\mathscr{A}}$-essential subcomplexes follows from a generalization of Proposition 1.4 stated in Proposition 2.4. We give more details in Section 2, where Theorem 1.3 is restated and proved in the more general setting of oriented matroids.

The first main result in this paper is the construction of the minimal free resolution of an arbitrary matroid ideal (see Theorems 3.3 and 3.9) and an arbitrary 
oriented matroid ideal (see Theorem 2.2). A numerical consequence of this result is a refinement of Stanley's formula, given in [16, Th. 9], for their Betti numbers (see Corollaries 2.3 and 3.4; see also the last paragraph of Section 3). The simplicial complexes corresponding to matroid ideals and oriented matroid ideals are the complexes of independent sets in matroids (see Remark 3.1) and the triangulations of Lawrence matroid polytopes (see Theorem 2.9), respectively. In the unimodular case, oriented matroid ideals arise as initial ideals of toric varieties in $\mathbb{P}^{1} \times \mathbb{P}^{1} \times \cdots \times \mathbb{P}^{1}$, by work of Bayer, Popescu, and Sturmfels [3, §4], and their Betti numbers can be interpreted as face numbers of hyperplane arrangements on a torus (see Theorem 4.1). Every ideal considered in this paper is Cohen-Macaulay; its Cohen-Macaulay type (highest Betti number) is the Möbius invariant of the underlying matroid, and all other Betti numbers are sums of Möbius invariants of matroid minors (see Section 4 and (8)).

Our second main result concerns the minimal free resolutions for graphic and cographic matroid ideals. In Section 5 we resolve the enumerative problems that were left open in [3, §5]. Propositions 5.3 and 5.7 give combinatorial expressions for the Möbius invariant of any graph. More precise and explicit formulas, in terms of Hermite polynomials, are established for the Möbius coinvariants of complete graphs (see Theorem 5.8) and of complete bipartite graphs (see Theorem 5.14).

\section{Oriented matroid ideals}

In this section we establish a link between oriented matroids and commutative algebra. In the resulting combinatorial context, the algebraists' classic question, "What makes a complex exact?" (see [7]), receives a surprising answer: it is the topological representation theorem of J. Folkman and J. Lawrence (see [6, Chap. 5]).

We start by briefly reviewing one of the axiom systems for oriented matroids (see [6]). Fix a finite set $E$. A sign vector $X$ is an element of $\{+,-, 0\}^{E}$. The positive part of $X$ is denoted $X^{+}=\left\{i \in E: X_{i}=+\right\}$, and $X^{-}$and $X^{0}$ are defined similarly. The support of $X$ is $X=\left\{i \in E: X_{i} \neq 0\right\}$. The opposite $-X$ of a vector $X$ is given by $(-X)_{i}=-X_{i}$. The composition $X \circ Y$ of two vectors $X$ and $Y$ is the sign vector defined by

$$
(X \circ Y)_{i}= \begin{cases}X_{i} & \text { if } X_{i} \neq 0 \\ Y_{i} & \text { if } X_{i}=0 .\end{cases}
$$

The separation set of sign vectors $X$ and $Y$ is $S(X, Y)=\left\{i \in E \mid X_{i}=-Y_{i} \neq 0\right\}$.

A set $\mathscr{L} \subseteq\{+,-, 0\}^{E}$ is the set of covectors of an oriented matroid on $E$ if and only if it satisfies the following four axioms (see [6, § 4.1.1]):

(1) the zero sign vector zero is in $\mathscr{L}$;

(2) $\quad$ if $X \in \mathscr{L}$, then $-X \in \mathscr{L}$ (symmetry);

(3) if $X, Y \in \mathscr{L}$, then $X \circ Y \in \mathscr{L}$ (composition); 
if $X, Y \in \mathscr{L}$ and $i \in S(X, Y)$, then there exists $Z \in \mathscr{L}$ such that $Z_{i}=0$ and $Z_{j}=(X \circ Y)_{j}=(Y \circ X)_{j}$ for all $j \notin S(X, Y)$ (elimination).

Somewhat informally, we say that such a pair $(E, \mathscr{L})$ is an oriented matroid. An affine oriented matroid (see [6, §10.1]), denoted $\mathscr{M}=(E, \mathscr{L}, g)$, is an oriented matroid with a distinguished element $g \in E$ such that $g$ is not a loop; that is, $X_{g} \neq 0$ for at least one covector $X \in \mathscr{L}$. The positive part of $\mathscr{L}$ is $\mathscr{L}^{+}=\left\{X \in \mathscr{L}: X_{g}=+\right\}$.

The set $\{+,-, 0\}^{E}$ is partially ordered by the product of partial orders

$$
0<+\quad \text { and } \quad 0<-\quad(+ \text { and }- \text { are not comparable }) \text {. }
$$

This induces a partial order on the set of covectors $\mathscr{L}$. A covector $X$ is called bounded if every nonzero covector $Y \leq X$ is in the positive part $\mathscr{L}^{+}$.

The topological representation theorem for oriented matroids (see [6, Th. 5.2.1]) states that $\widehat{\mathscr{L}}=\mathscr{L} \cup\{\hat{1}\}$ is the face lattice of an arrangement of pseudospheres; and $\widehat{\mathscr{L}}^{+}=\mathscr{L}^{+} \cup\{\hat{0}, \hat{1}\}$ is the face lattice of an arrangement of pseudohyperplanes (see [6, Exer. 5.8]). These are regular CW-complexes homeomorphic to a sphere and a ball, respectively. (This is why $\widehat{\mathscr{L}}$ is called the face lattice, and $\widehat{\mathscr{L}}^{+}$is called the affine face lattice, of $\mathscr{M}$.) The bounded complex $B_{\mathscr{M}}$ of $\mathscr{M}$ is their subcomplex formed by the cells associated with the bounded covectors. The bounded complex is uniquely determined by its face lattice-the poset of bounded covectors. Slightly abusing notation, we denote this poset by the same symbol, $B \mathscr{M}$.

We write $\mathrm{rk}(\cdot)$ for the rank function of the lattice $\widehat{\mathscr{L}}$. The atoms of $\widehat{\mathscr{L}}$, that is, the elements of rank 1 , are called cocircuits of $\mathscr{M}$. The vertices of the bounded complex $B_{\mathscr{M}}$ are exactly the cocircuits of $\mathscr{M}$ which belong to the positive part $\mathscr{L}^{+}$.

\section{Example 2.1 (Affine oriented matroids from hyperplane arrangements)}

Let $\mathscr{C}=\left\{H_{1}, \ldots, H_{n}, H_{g}\right\}$ be a central hyperplane arrangement in $\mathbb{R}^{d+1}=\mathbb{R}^{d} \times \mathbb{R}$, written as $H_{i}=\left\{(v, w) \in \mathbb{R}^{d} \times \mathbb{R}: h_{i}(v)=c_{i} w\right\}$ and $H_{g}=\{(v, w): w=0\}$. The restriction of $\mathscr{C}$ to the hyperplane $\{(v, w): w=1\}$ is precisely the affine arrangement $\mathscr{A}$ in Section 1. Fix $E=\{1, \ldots, n, g\}$. The image of the map

$$
\begin{aligned}
\mathbb{R}^{d+1} & \rightarrow\{+,-, 0\}^{E}, \\
(v, w) & \mapsto\left(\operatorname{sign}\left(h_{1}(v)-c_{1} w\right), \ldots, \operatorname{sign}\left(h_{n}(v)-c_{n} w\right), \operatorname{sign}(w)\right)
\end{aligned}
$$

is the set $\mathscr{L}$ of covectors of an oriented matroid on $E$. The affine face lattice $\widehat{\mathscr{L}}^{+}$of $\mathscr{M}=(E, \mathscr{L}, g)$ equals the face lattice of the affine hyperplane arrangement $\mathscr{A}$. The bounded complex $B_{\mathscr{M}}$ coincides with the bounded complex $B_{\mathscr{A}}$ in Proposition 1.4.

Let $\mathscr{M}=(E, \mathscr{L}, g)$ be an affine oriented matroid on $E=\{1, \ldots, n, g\}$. With every sign vector $Z \in\{0,+,-\}^{E}$, we associate a monomial

$$
m_{x y}(Z)=\left(\prod_{i: Z_{i}=+} x_{i}\right) \cdot\left(\prod_{i: Z_{i}=-} y_{i}\right), \quad \text { where } x_{g}=y_{g}=1 \text {. }
$$


The oriented matroid ideal $O$ is the ideal in the polynomial $\operatorname{ring} \mathbf{k}[\mathbf{x}, \mathbf{y}]=\mathbf{k}\left[x_{1}, \ldots\right.$, $x_{n}, y_{1}, \ldots, y_{n}$ ] generated by all monomials corresponding to covectors $Z \in \mathscr{L}^{+}$. The matroid ideal $M$ associated with $\mathscr{M}=(E, \mathscr{L}, g)$ is the ideal of $\mathbf{k}[\mathbf{x}]$ obtained from $O$ by specializing $y_{i}$ to $x_{i}$ for all $i$. These ideals are treated in Section 3. The main result of this section concerns the syzygies of the oriented matroid ideal $O$.

\section{THEOREM 2.2}

The oriented matroid ideal $O$ is minimally generated by the monomials corresponding to the vertices of $B \mathscr{M}$. The bounded complex $B \mathscr{M}$ is an $O$-complex. Thus its cellular complex $C_{\bullet}\left(B_{\mathscr{M}}, O\right)$ gives a minimal $\mathbb{N}^{2 n}$-graded free $\mathbf{k}[\mathbf{x}, \mathbf{y}]$-resolution of $O$.

Recall that, for a monomial $m$ in $\mathbf{k}[\mathbf{x}, \mathbf{y}]$, the corresponding $\mathbb{N}^{2 n}$-graded Betti number of $O, \beta_{m}(O)$ is the multiplicity of the summand $\langle m\rangle$ in a minimal $\mathbb{N}^{2 n}$-graded $\mathbf{k}[\mathbf{x}, \mathbf{y}]$ resolution of $O$. Theorem 2.2 implies the following numerical result.

\section{COROLLARY 2.3}

The $\mathbb{N}^{2 n}$-graded Betti numbers of $O$ are all 0 or 1 . They are given by the coefficients in the numerator of the $\mathbb{N}^{2 n}$-graded Hilbert series of $O$ :

$$
\left(\sum_{Z \in B_{\mathscr{M}}}(-1)^{\mathrm{rk}(Z)} m_{x y}(Z)\right) / \prod_{i=1}^{n}\left(1-x_{i}\right)\left(1-y_{i}\right) .
$$

\section{Proof of Theorem 2.2}

Distinct cells $Z$ and $Z^{\prime}$ of the bounded complex $B_{\mathscr{M}}$ have distinct labels: $m_{x y}(Z) \neq$ $m_{x y}\left(Z^{\prime}\right)$. This implies minimality of the complex $C_{\bullet}\left(B_{\mathscr{M}}, O\right)$. In order to prove exactness of $C_{\bullet}\left(B_{\mathscr{M}}, O\right)$, we must verify the first hypothesis in Proposition 1.1. To this end, we shall digress and first present a generalization of Proposition 1.4.

The regions of an oriented matroid $(E, \mathscr{L})$ are the maximal covectors, that is, the maximal elements of the poset $\mathscr{L}$. For a covector $X \in \mathscr{L}$ and a subset $E^{\prime}$ of $E$, denote by $\left.X\right|_{E^{\prime}} \in\{+,-, 0\}^{E^{\prime}}$ the restriction of $X$ to $E^{\prime}:\left(\left.X\right|_{E^{\prime}}\right)_{i}=X_{i}$ for every $i \in E^{\prime}$. The restriction of $(E, \mathscr{L})$ to a subset $E^{\prime}$ of $E$ is the oriented matroid on $E^{\prime}$ with the set of covectors $\left.\mathscr{L}\right|_{E^{\prime}}=\left\{\left.X\right|_{E^{\prime}}: X \in \mathscr{L}\right\}$.

The following result, which was cited without proof in [3, Th. 4.4], is implicit in the derivation of [6, Th. 4.5.7]. We are grateful to G. Ziegler for making this explicit by showing us the following proof. Ziegler's proof does not rely on the topological representation theorem for oriented matroids. If one uses that theorem, then the following proposition can also be proved by a topological argument. 
plex. For any subset $E^{\prime}$ of $E$ and any region $R^{\prime}$ of $\left(E^{\prime},\left.\mathscr{L}\right|_{E^{\prime}}\right)$, the $C W$-complex with the face poset $B^{\prime}=\left\{X \in B \mathscr{M}:\left.X\right|_{E^{\prime}} \leq R^{\prime}\right\}$ is contractible.

\section{Proof}

Let $\mathbb{T}$ denote the set of regions of $\mathscr{L}$. A subset $A \subseteq \mathbb{T}$ is said to be $T$-convex if it is an intersection of "half-spaces," that is, sets of the form $\mathbb{T}_{e}^{+}=\left\{T \in \mathbb{T}: T_{e}=+\right\}$ and $\mathbb{T}_{e}^{-}=\left\{T \in \mathbb{T}: T_{e}=-\right\}$. Each region $R \in \mathbb{T}$ defines a partial order on $\mathbb{T}$ :

$$
T_{1} \leq T_{2}: \Longleftrightarrow\left\{e \in E: R_{e}=-\left(T_{1}\right)_{e}\right\} \subseteq\left\{e \in E: R_{e}=-\left(T_{2}\right)_{e}\right\} .
$$

Denote this poset by $\mathbb{T}(\mathscr{L}, R)$. We also abbreviate $\mathbb{T}^{+}:=\mathbb{T}_{g}^{+}=\mathbb{T} \cap \mathscr{L}^{+}$.

We may assume that $B^{\prime}$ is nonempty. Then $\mathscr{R}:=\left\{X \in \mathbb{T}^{+}:\left.X\right|_{E^{\prime}}=R^{\prime}\right\}$ is a nonempty, $T$-convex set. It is stated in [6, Lem. 4.5.5] that $\mathscr{R}$ is an order ideal of $\mathbb{T}(\mathscr{L}, R)$, and, moreover, it is an order ideal of $\mathbb{T}^{+} \subseteq \mathbb{T}(\mathscr{L}, R)$. By [6, Prop. 4.5.6], there exists a recursive coatom ordering of $\widehat{\mathscr{L}}^{+}$in which the elements of $\mathscr{R}$ come first. The restriction of this ordering to $\mathscr{R}$ is a recursive coatom ordering of the poset $\widehat{\mathscr{L}}_{\mathscr{R}}^{+}=\left\{X \in \mathscr{L}^{+}: X \leq T\right.$ for some $\left.T \in \mathscr{R}\right\} \cup\{\hat{1}\}$. This implies (using [6, Lem. 4.7.18]) that the order complex $\Delta_{\text {ord }}\left(\mathscr{L}_{\mathscr{R}}^{+}\right)$of $\mathscr{L}_{\mathscr{R}}^{+}$is a shellable $(r-1)$-ball. It is a subcomplex of $\Delta_{\text {ord }}\left(\mathscr{L}^{+}\right)$, which is also an $(r-1)$-ball, by [6, Th. 4.5.7]. Let $U=\mathscr{L}_{\mathscr{R}}^{+} \backslash B_{\mathscr{M}}$ be the set of "unbounded covectors." Then the subcomplex $\Delta_{U}$ of $\Delta_{\text {ord }}\left(\mathscr{L}_{\mathscr{R}}^{+}\right)$induced on the vertex set of $U$ lies in the boundary of $\Delta_{\text {ord }}\left(\mathscr{L}^{+}\right)$and hence also in the boundary of $\Delta_{\text {ord }}\left(\mathscr{L}_{\mathscr{R}}^{+}\right)$. Thus $\left\|\Delta_{\text {ord }}\left(\mathscr{L}_{\mathscr{R}}^{+}\right)\right\| \backslash\left\|\Delta_{U}\right\|$ is a contractible space. By [6, Lem. 4.7.27], the space $\left\|\Delta_{\text {ord }}\left(B^{\prime}\right)\right\|$ is a strong deformation retract of $\left\|\Delta_{\text {ord }}\left(\mathscr{L}_{\mathscr{R}}^{+}\right)\right\| \backslash\left\|\Delta_{U}\right\|$ and is hence contractible as well.

We now finish the proof of Theorem 2.2. Consider any $O$-essential subcomplex $(B \mathscr{M})_{\leq \mathbf{x}^{\mathbf{a}} \mathbf{y}} \mathbf{b}$ of $B \mathscr{M}$, with $\mathbf{a}, \mathbf{b} \in \mathbb{N}^{n}$. This complex consists of all cells $Z$ whose label $m_{x y}(Z)$ divides $\mathbf{x}^{\mathbf{a}} \mathbf{y}^{\mathbf{b}}$. Set

$$
\begin{aligned}
E^{\prime \prime} & =\left\{1 \leq i \leq n: a_{i}=0 \text { and } b_{i}=0\right\}, \\
E^{\prime} & =\left\{1 \leq i \leq n: \text { exactly one of } a_{i} \text { and } b_{i} \text { is positive }\right\} \subseteq E \backslash E^{\prime \prime} .
\end{aligned}
$$

We first replace our affine oriented matroid $(E, \mathscr{L}, g)$ by the affine oriented matroid $\left(E \backslash E^{\prime \prime}, \mathscr{L} / E^{\prime \prime}, g\right)$ gotten by contraction at $E^{\prime \prime}$. Next we define $R^{\prime} \in\{+,-, 0\}^{E^{\prime}}$ by

$$
R_{i}^{\prime}=\left\{\begin{array}{l}
+ \text { if } a_{i}>0, \\
-\quad \text { if } b_{i}>0,
\end{array} \text { for every } i \in E^{\prime} .\right.
$$

We apply Proposition 2.4 with this $R^{\prime}$ to $\left(E \backslash E^{\prime \prime}, \mathscr{L} / E^{\prime \prime}, g\right)$. Then $B^{\prime}$ is the face poset of $(B \mathscr{M})_{\leq \mathbf{x}^{\mathbf{a}} \mathbf{y}} \mathbf{b}$, which is therefore contractible.

The oriented matroid ideal $O$ is squarefree and hence is the Stanley-Reisner ideal of a simplicial complex $\Delta \mathscr{M}$ on $2 n$ vertices $\left\{1, \ldots, n, 1^{\prime}, \ldots, n^{\prime}\right\}$, whose faces correspond 
to squarefree monomials of $\mathbf{k}[\mathbf{x}, \mathbf{y}]$ which do not belong to $O$; that is,

$$
\left\{i_{1}, \ldots, i_{k}, j_{1}^{\prime}, \ldots, j_{m}^{\prime}\right\} \in \Delta \mathscr{M} \quad \text { if and only if } x_{i_{1}} \cdots x_{i_{k}} y_{j_{1}} \cdots y_{j_{m}} \notin O .
$$

In what follows we give a geometric description of that simplicial complex.

LEMMA 2.5

We have $F \cap\left\{i, i^{\prime}\right\} \neq \emptyset$ for any facet $F$ of $\Delta_{\mathscr{M}}$ and $i \in\{1, \ldots, n\}$.

\section{Proof}

Let $F$ be a face of $\Delta \mathscr{M}$ such that $F \cap\left\{i, i^{\prime}\right\}=\emptyset$. Suppose that neither $F^{\prime}=F \cup\{i\}$ nor $F^{\prime \prime}=F \cup\left\{i^{\prime}\right\}$ is a face of $\Delta_{\mathscr{M}}$. Then there exist cocircuits $Z^{\prime}, Z^{\prime \prime} \in B_{\mathscr{M}}$ such that

$$
\begin{array}{ll}
Z_{i}^{\prime}=+, & \left(Z^{\prime}\right)^{+} \backslash\{i\} \subseteq\{1 \leq j \leq n: j \in F\} \cup\{g\}, \\
& \left(Z^{\prime}\right)^{-} \subseteq\left\{1 \leq j \leq n: j^{\prime} \in F\right\}, \\
Z_{i}^{\prime \prime}=-, & \left(Z^{\prime \prime}\right)^{+} \subseteq\{1 \leq j \leq n: j \in F\} \cup\{g\}, \\
& \left(Z^{\prime \prime}\right)^{-} \backslash\{i\} \subseteq\left\{1 \leq j \leq n: j^{\prime} \in F\right\} .
\end{array}
$$

By the strong elimination axiom applied to $\left(Z^{\prime}, Z^{\prime \prime}, i, g\right)$, there is a cocircuit $Z$ such that $Z_{i}=0, \quad Z_{g}=+, \quad Z^{+} \subseteq\left(Z^{\prime}\right)^{+} \cup\left(Z^{\prime \prime}\right)^{+}, \quad Z^{-} \subseteq\left(Z^{\prime}\right)^{-} \cup\left(Z^{\prime \prime}\right)^{-}$. Thus $Z \in B_{\mathscr{M}}$, and the monomial $m_{x y}(F)$ is divisible by $m_{x y}(Z) \in O$. This contradicts $F \in \Delta_{\mathscr{M}}$.

Suppose now that the affine oriented matroid $\mathscr{M}=(E, \mathscr{L}, g)$ is a single-element extension of the matroid $\mathscr{M} \backslash g=(E \backslash g, \mathscr{L} \backslash g)$ by an element $g$ in general position, in the sense of [6, Prop. 7.2.2]. For the affine arrangement $\mathscr{A}$ in Section 1 or Example 2.1 , this means that $\mathscr{A}$ has no vertices at infinity. In such a case, Theorem 2.2 implies the following properties of $O$. We denote by $r$ the rank of $\mathscr{M} \backslash g$.

COROLLARY 2.6

The ring $\mathbf{k}\left[\Delta_{\mathscr{M}}\right]=\mathbf{k}[\mathbf{x}, \mathbf{y}] / O$ is a Cohen-Macaulay ring of dimension $2 n-r$.

\section{Proof}

Since $\operatorname{rk}(\mathscr{M} \backslash g)=r$, every $(n-r+1)$-element subset $\left\{i_{1}, \ldots, i_{n-r+1}\right\}$ of $\{1, \ldots, n\}$ contains the support of a (signed) cocircuit. This implies that every monomial of the form $x_{i_{1}} \cdots x_{i_{n-r+1}} y_{i_{1}} \cdots y_{i_{n-r+1}}$ belongs to $O$. The variety defined by these monomials is a subspace arrangement of codimension $r$. Hence $O$ has codimension greater than or equal to $r$, which means that the ring $\mathbf{k}\left[\Delta_{\mathscr{M}}\right]=\mathbf{k}[\mathbf{x}, \mathbf{y}] / O$ has Krull dimension less than or equal to $2 n-r$. By Theorem 2.2, the bounded complex $B_{\mathscr{M}}$ supports 
a minimal free resolution of $O$, and therefore

$$
\operatorname{depth}\left(\mathbf{k}\left[\Delta_{\mathscr{M}}\right]\right)=2 n-(\text { the length of this resolution })=2 n-r .
$$

Hence $\operatorname{depth}\left(\mathbf{k}\left[\Delta_{\mathscr{M}}\right]\right)=\operatorname{dim}\left(\mathbf{k}\left[\Delta_{\mathscr{M}}\right]\right)=2 n-r$, and $\mathbf{k}\left[\Delta_{\mathscr{M}}\right]$ is Cohen-Macaulay.

The result in Corollary 2.6 can be strengthened to the statement that the simplicial complex $\Delta_{M}$ is shellable. This follows from Theorem 2.9.

\section{COROLLARY 2.7}

The set $\left\{x_{1}-y_{1}, \ldots, x_{n}-y_{n}\right\}$ is a regular sequence on $\mathbf{k}\left[\Delta_{\mathscr{M}}\right]=\mathbf{k}[\mathbf{x}, \mathbf{y}] / O$.

\section{Proof}

Since $\mathbf{k}\left[\Delta_{\mathscr{M}}\right]$ is Cohen-Macaulay, it suffices to show that $\left\{x_{1}-y_{1}, \ldots, x_{n}-y_{n}\right\}$ is a part of a linear system of parameters (1.s.o.p.). This follows from Lemma 2.5 and the 1.s.o.p. criterion due to B. Kind and P. Kleinschmidt [19, Lem. III.2.4].

Consider any signed circuit $C=\left(C^{+}, C^{-}\right)$of our oriented matroid such that $g$ lies in $C^{-}$. By the general position assumption on $g$, the complement of $g$ in that circuit is a basis of the underlying matroid. We write $P_{C}$ for the ideal generated by the variables $x_{i}$ for each $i \in C^{+}$and by the variables $y_{j}$ for each $j \in C^{-} \backslash\{g\}$.

\section{PROPOSITION 2.8}

The minimal prime decomposition of the oriented matroid ideal equals $O=\bigcap_{C} P_{C}$, where the intersection is over all circuits $C$ such that $g \in C^{-}$.

\section{Proof}

The right-hand side is easily seen to contain the left-hand side. For the converse it suffices to divide by the regular sequence $x_{1}-y_{1}, \ldots, x_{n}-y_{n}$ and note that the resulting decomposition for the matroid ideal $M$ is easy (see Remark 3.1).

Our final result relates the ideal $O$ to matroid polytopes and their triangulations. The monograph of F. Santos [15] provides an excellent state-of-the-art introduction. We refer in particular to [15, §4], where Santos introduces triangulations of Lawrence (matroid) polytopes, and he shows that these are in bijection with one-element liftings of the underlying matroid. Under matroid duality, one-element liftings correspond to one-element extensions. In our context these extensions correspond to adding the special element $g$, which plays the role of the pseudohyperplane at infinity. From Santos's result we infer the following theorem. 


\section{THEOREM 2.9}

The oriented matroid ideal $O$ is the Stanley-Reisner ideal of the triangulation of the Lawrence matroid polytope induced by the lifting dual to the extension by g. In particular, $O$ is the Stanley-Reisner ideal of a triangulated ball.

The second assertion holds because lifting triangulations of matroid polytopes are triangulated balls and, by Santos's work, every triangulation of a Lawrence matroid polytope is a lifting triangulation. We remark that it is unknown whether arbitrary triangulations of matroid polytopes are topological balls (see [15, p. 7]).

\section{Matroid ideals}

Let $\underline{M}$ be an (unoriented) matroid on the set $\{1, \ldots, n\}$, and let $L$ be its lattice of flats. We encode $\underline{M}$ by the matroid ideal $M$ generated by the monomials $m_{x}(F)=\prod_{i: i \notin F} x_{i}$ for every proper flat $F \in L$. The minimal generators of $M$ are the squarefree monomials representing cocircuits of $\mathscr{M}$, that is, the monomials $m_{x}(H)$, where $H$ runs over all hyperplanes of $\mathscr{M}$. Equivalently, $M$ is the StanleyReisner ideal of the simplicial complex of independent sets of the dual matroid $\mathscr{M}^{*}$. The following explains what happens when we substitute $y_{i} \mapsto x_{i}$ in Proposition 2.8.

\section{Remark 3.1}

The matroid ideal $M$ has the minimal prime decomposition

$$
M=\bigcap_{B \text { basis of } \underline{\underline{M}}}\left\langle x_{i} \mid i \in B\right\rangle .
$$

The following characterization of our ideals can serve as a definition of the word matroid. It is a translation of the (co)circuit axiom into commutative algebra.

\section{Remark 3.2}

A proper squarefree monomial ideal $M$ of $\mathbf{k}[\mathbf{x}]$ is a matroid ideal if and only if, for every pair of monomials $m_{1}, m_{2} \in M$ and any $i \in\{1, \ldots, n\}$ such that $x_{i}$ divides both $m_{1}$ and $m_{2}$, the monomial $1 \mathrm{~cm}\left(m_{1}, m_{2}\right) / x_{i}$ is in $M$ as well.

Matroid ideals have been studied since the earliest days of combinatorial commutative algebra as a paradigm for shellability and Cohen-Macaulayness. Stanley computed their Betti numbers in [16, Th. 9]. The purpose of this section is to construct an explicit minimal $\mathbf{k}[\mathbf{x}]$-free resolution for any matroid ideal $M$. We note that in recent work of V. Reiner and Welker [14] the term "matroid ideal" is used for the squarefree monomial ideals that are Alexander dual to our matroid ideals. 
We first consider the case where $\mathscr{M}$ is an orientable matroid. This means that there exists an oriented matroid $\mathscr{M}$ whose underlying matroid is $\mathscr{M}$. Let $\mathscr{L}$ be the set of covectors of a single element extension of $\mathscr{M}$ by an element $g$ in general position (see [6, Prop. 7.2.2]). Consider the affine oriented matroid $\widetilde{\mathscr{M}}=(E, \mathscr{L}, g)$, where $E=\{1, \ldots, n\} \cup\{g\}$, and its bounded complex $B \tilde{\mathscr{M}}$. Note that, for each sign vector $Z$ in $B \widetilde{\mathscr{M}}$, the zero set $Z^{0}$ is a flat in $L$. Moreover, by the genericity hypothesis on $g$, all flats arise in this way. We label each cell $Z$ of the bounded complex $B \widetilde{\mathscr{M}}$ by the monomial $m_{x}(Z)=\prod\left\{x_{i}: 1 \leq i \leq n\right.$ and $\left.Z_{i} \neq 0\right\}$.

\section{THEOREM 3.3}

Let $M$ be the matroid ideal of an orientable matroid. Then the bounded complex $B \tilde{\mathscr{M}}$ of any corresponding affine oriented matroid is an $M$-complex, and its cellular complex $C_{\bullet}(B \tilde{\mathscr{M}}, M)$ gives a minimal free resolution of $M$ over $\mathbf{k}[\mathbf{x}]$.

\section{Proof}

Let $\mathbf{a}=\left(a_{1}, \ldots, a_{n}\right) \in \mathbb{N}^{n}$, and consider the $M$-essential subcomplex $(B \widetilde{\mathscr{M}})_{\leq \mathbf{x}} \mathbf{a}$. This complex (if not empty) is the bounded complex of the contraction of $(E, \mathscr{L}, g)$ by $\left\{1 \leq i \leq n: a_{i}=0\right\}$ and hence is acyclic by Proposition 2.4. Since $m_{x}\left(Z^{\prime}\right)$ is a proper divisor of $m_{x}(Z)$ whenever $Z^{\prime}<Z$ and $Z^{\prime}, Z \in B_{\tilde{M}}$, it follows that $B \widetilde{\mathscr{M}}^{\text {is }}$ an $M$-complex.

We remark that $C_{\bullet}\left(B_{\widetilde{M}}, M\right)$ is obtained from the complex $C_{\bullet}\left(B_{\mathscr{M}}, O\right)$, where $O$ is the oriented matroid ideal of $\widetilde{\mathscr{M}}=(E, \mathscr{L}, g)$, by specializing $y_{i}$ to $x_{i}$ for all $i$. Hence Theorem 2.2 and Corollary 2.7 give a second proof of Theorem 3.3.

COROLLARY 3.4

The $\mathbb{N}^{n}$-graded Hilbert series of any matroid ideal $M$ equals

$$
\left(\sum_{F \in L} \mu_{L}(F, \hat{1}) \cdot \prod\left\{x_{j}: j \notin F\right\}\right) / \prod_{i=1}^{n}\left(1-x_{i}\right),
$$

where $L$ is the lattice of flats of $\underline{\mathscr{M}}$, and $\mu_{L}$ is its Möbius function.

There are several ways of deriving this corollary. First, it follows from [16, Th. 9]. A second possibility is to observe that the geometric lattice $L$ coincides with the lcm lattice (in the sense of [8]) of the ideal $M$, and then [8, Th. 2.1] implies the claim. Finally, in the orientable case, Corollary 3.4 follows from Theorem 3.3 and the oriented matroid version of T. Zaslavksy's face-count formula. 
PROPOSITION 3.5 (Zaslavsky's formula (see [22], [6, Th. 4.6.5]))

The number of bounded regions of a rank $r$ affine oriented matroid $\widetilde{\mathscr{M}}=(E, \mathscr{L}, g)$ equals $(-1)^{r} \mu_{L}(\hat{0}, \hat{1})$.

We next treat the case of nonorientable matroids. It would be desirable to construct an $M$-complex for an arbitrary matroid ideal $M$ and to explore the "space" of all possible $M$-complexes. Currently we do not know how to construct them. Therefore we introduce a different technique for resolving $M$ minimally.

Let $P$ be any graded poset that has a unique minimal element $\hat{0}$ and a unique maximal element $\hat{1}$. (Later on, we take $P$ to be the order dual of our geometric lattice $L$.) Let $\Delta(P)$ denote the order complex of $P$, that is, the simplicial complex whose simplices $\left[F_{0}, F_{1}, \ldots, F_{i}\right]$ are decreasing chains $\hat{1}>F_{0}>F_{1}>\cdots>F_{i}>\hat{0}$. For $F \in P$, denote by $\Delta(F)$ the order complex of the lower interval $[\hat{0}, F]$. Note that $\operatorname{dim} \Delta(F)=\operatorname{rk}(F)-2$. Let $C_{i}(\Delta(F))$ be the $\mathbf{k}$-vector space of $i$-dimensional chains of $\Delta(F)$, and let

$$
\begin{gathered}
0 \longrightarrow C_{\mathrm{rk}(F)-2}(\Delta(F)) \longrightarrow \cdots \stackrel{\partial_{2}}{\longrightarrow} C_{1}(\Delta(F)) \\
\stackrel{\partial_{1}}{\longrightarrow} C_{0}(\Delta(F)) \stackrel{\partial_{0}}{\longrightarrow} C_{-1}(\Delta(F)) \longrightarrow 0
\end{gathered}
$$

be the usual (augmented) chain complex; that is, the differential is given by

$$
\partial_{i}\left[F_{0}, F_{1}, \ldots, F_{i}\right]=\sum_{j=0}^{i}(-1)^{j}\left[F_{0}, \ldots, \widehat{F_{j}}, \ldots, F_{i}\right] \quad \text { for } i>0 \text { and } \partial_{0}\left[F_{0}\right]=0 .
$$

Denote by $Z_{i}(\Delta(F))=\operatorname{ker}\left(\partial_{i}\right)$ the space of $i$-cycles, and by $\widetilde{H}_{i}(\Delta(F))$ the $i$ th (reduced) homology of $\Delta(F)$. (For relevant background on poset homology, see [5].)

For each pair $F, F^{\prime} \in P$ such that $\operatorname{rk}(F)-\operatorname{rk}\left(F^{\prime}\right)=1$, we define a map

$$
\phi: C_{i}(\Delta(F)) \longrightarrow C_{i-1}\left(\Delta\left(F^{\prime}\right)\right)
$$

by

$$
\left[F_{0}, F_{1}, \ldots, F_{i}\right] \mapsto \begin{cases}0 & \text { if } F_{0} \neq F^{\prime} \\ {\left[F_{1}, \ldots, F_{i}\right]} & \text { if } F_{0}=F^{\prime} .\end{cases}
$$

The map $\phi$ is zero unless $F^{\prime} \lessdot F$ (in other words, $F$ covers $F^{\prime}$ ). Note that $\partial \circ \phi=$ $-\phi \circ \partial$, and hence the restriction of $\phi$ to cycles gives a map $\phi: Z_{i}(\Delta(F)) \longrightarrow$ $Z_{i-1}\left(\Delta\left(F^{\prime}\right)\right)$. Combining these maps, we obtain a complex of $\mathbf{k}$-vector spaces:

$$
\begin{aligned}
\mathscr{Z}(P): & 0 \longrightarrow Z_{r-2}(\Delta(P)) \stackrel{\phi}{\longrightarrow} \underset{\mathrm{rk}(F)=r-1}{\bigoplus} Z_{r-3}(\Delta(F)) \\
& \stackrel{\phi}{\longrightarrow} \cdots \stackrel{\phi}{\longrightarrow} \bigoplus_{\mathrm{rk}(F)=2} Z_{0}(\Delta(F)) \stackrel{\phi}{\longrightarrow} \bigoplus_{\mathrm{rk}(F)=1} Z_{-1}(\Delta(F)) \longrightarrow \mathbf{k} .
\end{aligned}
$$


(Here $\Delta(P)$ is regarded as $\Delta(\hat{1})$, and thus the first map $\phi$ is well defined.) The complex property $\phi^{2}=0$ is verified by direct calculation using equation (4). Let $P_{(j)}$ denote the poset obtained from $P$ by removing all rank levels greater than or equal to $j$, and let $\Delta\left(P_{(j)}\right)$ be the order complex of $P_{(j)} \cup\{\hat{1}\}$.

\section{PROPOSITION 3.6}

The complex $\mathscr{Z}(P)$ is exact if $\widetilde{H}_{i}\left(\Delta\left(P_{(i+3)}\right)\right)=0$ for all $i \leq r-3$.

To prove Proposition 3.6 we need some notation. If $x \in \bigoplus_{\mathrm{rk}(F)=i} Z_{i-2}(\Delta(F))$, we denote its $F$-component by $x_{F}$. For a simplex $\sigma=\left[F_{0}, F_{1}, \ldots, F_{i}\right]$, we also write $\sigma=F_{0} *\left[F_{1}, \ldots, F_{i}\right]$, and the operation “*” extends to k-linear combinations.

\section{Remark 3.7}

Suppose that $z \in C_{i}\left(\Delta\left(P_{(i+2)}\right)\right)$. Then $z$ can be expressed as

$$
z=\sum_{\operatorname{rk}\left(F^{\prime}\right)=i+1} F^{\prime} * y_{F^{\prime}}=\sum_{\operatorname{rk}\left(F^{\prime}\right)=i+1} \sum_{F^{\prime \prime} \lessdot F^{\prime}} F^{\prime} * F^{\prime \prime} * x_{F^{\prime}}, F^{\prime \prime},
$$

where $y_{F^{\prime}} \in C_{i-1}\left(\Delta\left(F^{\prime}\right)\right)$ and $x_{F^{\prime}, F^{\prime \prime}} \in C_{i-2}\left(\Delta\left(F^{\prime \prime}\right)\right)$. Its boundary equals

$$
\begin{aligned}
\partial(z)= & \sum_{\operatorname{rk}\left(F^{\prime \prime}\right)=i} F^{\prime \prime} * \sum_{F^{\prime}>F^{\prime \prime}} x_{F^{\prime}, F^{\prime \prime}} \\
& -\sum_{\operatorname{rk}\left(F^{\prime}\right)=i+1} F^{\prime} * \sum_{F^{\prime \prime} \lessdot F^{\prime}} x_{F^{\prime}, F^{\prime \prime}}+\sum_{F^{\prime}, F^{\prime \prime}} F^{\prime} * F^{\prime \prime} * \partial\left(x_{F^{\prime}, F^{\prime \prime}}\right) .
\end{aligned}
$$

We conclude that $z$ is a cycle if and only if the following conditions are satisfied:

$$
\begin{aligned}
\sum_{F^{\prime} \gg F^{\prime \prime}} x_{F^{\prime}, F^{\prime \prime}}=0 & \text { for all } F^{\prime \prime} \text { with } \operatorname{rk}\left(F^{\prime \prime}\right)=i ; \\
\sum_{F^{\prime \prime} \lessdot F^{\prime}} x_{F^{\prime}, F^{\prime \prime}}=0 & \text { for all } F^{\prime} \text { with } \operatorname{rk}\left(F^{\prime}\right)=i+1 ; \\
\partial\left(x_{F^{\prime}, F^{\prime \prime}}\right)=0 & \text { for all } F^{\prime}, F^{\prime \prime} \text { such that } F^{\prime \prime} \lessdot F^{\prime} .
\end{aligned}
$$

\section{Proof of Proposition 3.6}

To show that $\mathscr{Z}(P)$ is exact, consider $y=\left(y_{F^{\prime}}\right) \in \bigoplus_{\mathrm{rk}\left(F^{\prime}\right)=i+1} Z_{i-1}\left(\Delta\left(F^{\prime}\right)\right)$ such that $\phi(y)=0$. There are several cases. If $i=r-1$, then $y=y_{\hat{1}}$ can be expressed as $\sum_{\mathrm{rk}(F)=r-2} F * x_{F}$, where $x_{F} \in C_{r-3}(\Delta(F))$. Then $0=\phi(y)_{F}=x_{F}$, and therefore $y=0$. Hence the leftmost map $\phi$ is an inclusion.

Let $0<i<r-1$, and define $z=\sum_{\operatorname{rk}\left(F^{\prime}\right)=i+1} F^{\prime} * y_{F^{\prime}} \in C_{i}\left(\Delta\left(P_{(i+2)}\right)\right)$. We claim that $z$ is a cycle; that is, $z \in Z_{i}\left(\Delta\left(P_{(i+2)}\right)\right)$. Indeed, if $i>0$, then $y_{F^{\prime}}$ can be 
expressed as $\sum_{F^{\prime \prime} \lessdot F^{\prime}} F^{\prime \prime} * x_{F^{\prime}, F^{\prime \prime}}$, where $x_{F^{\prime}, F^{\prime \prime}} \in C_{i-2}\left(\Delta\left(F^{\prime \prime}\right)\right)$. Hence

$$
(\phi(y))_{F^{\prime \prime}}=\sum_{F^{\prime} \gg F^{\prime \prime}} x_{F^{\prime}, F^{\prime \prime}}, \quad \forall F^{\prime \prime} \text { with } \operatorname{rk}\left(F^{\prime \prime}\right)=i,
$$

and

$$
\partial\left(y_{F^{\prime}}\right)=\sum_{F^{\prime \prime} \lessdot F^{\prime}} x_{F^{\prime}, F^{\prime \prime}}-\sum_{F^{\prime \prime} \lessdot F^{\prime}} F^{\prime \prime} * \partial\left(x_{F^{\prime}, F^{\prime \prime}}\right), \quad \forall F^{\prime} \text { with } \operatorname{rk}\left(F^{\prime}\right)=i+1 .
$$

Since $\phi(y)=0$ and $\partial\left(y_{F^{\prime}}\right)=0$ for any $F^{\prime}$ of rank $i+1$, we infer that $z$ satisfies conditions (4) -(6) in Remark 3.7 and therefore is a cycle. In the case $i=0$, the proof is very similar. Now if $i=r-2$, then $z \in Z_{r-2}(\Delta(P))$, and $\phi(z)=$ $\phi\left(\sum F^{\prime} * y_{F^{\prime}}\right)=\left(y_{F^{\prime}}\right)=y$. Hence we are done in this case. If $i<r-2$, then, since $Z_{i}\left(\Delta\left(P_{(i+2)}\right)\right) \subseteq Z_{i}\left(\Delta\left(P_{(i+3)}\right)\right)$ and $\widetilde{H}_{i}\left(\Delta\left(P_{(i+3)}\right)\right)=0$, it follows that there exists $w \in C_{i+1}\left(\Delta\left(P_{(i+3)}\right)\right)$ such that $\partial(w)=z$. Express $w$ as $\sum_{\mathrm{rk}(F)=i+2} F * v_{F}$, where $v_{F} \in C_{i}(\Delta(F))$. Since $z=\partial(w)=\sum_{\mathrm{rk}(F)=i+2} v_{F}-\sum_{\mathrm{rk}(F)=i+2} F * \partial\left(v_{F}\right)$, we conclude that $\partial\left(v_{F}\right)=0$ for all $F$ of rank $i+2$ and that $\sum_{F} v_{F}=z=\sum_{F^{\prime}} F^{\prime} * y_{F^{\prime}}$. Thus $v=\left(v_{F}\right) \in \bigoplus_{\operatorname{rk}(F)=i+2} Z_{i}(\Delta(F))$, and $\phi(v)=y$.

\section{COROLLARY 3.8}

If $P$ is a Cohen-Macaulay poset, then $\mathscr{Z}(P)$ is exact.

\section{Proof}

If $\Delta(P)$ is Cohen-Macaulay, then $\Delta\left(P_{(i)}\right)$ is Cohen-Macaulay for every $i$ (see [17, Th. 4.3]). This means that all homologies of $\Delta\left(P_{(i)}\right)$ vanish, except possibly the top one. Thus the conditions of Proposition 3.6 are satisfied.

Suppose now that every atom $A$ of $P$ is labeled by a monomial $m_{A} \in \mathbf{k}[\mathbf{x}]$. The poset ideal $I_{P}$ is the ideal generated by these monomials. Associate with every element $F$ of $P$ a monomial $m_{F}$ as follows:

$$
m_{F}:=\operatorname{lcm}\left\{m_{A}: \operatorname{rk}(A)=1, A \leq F\right\} \quad \text { if } F \neq \hat{0} \text { and } m_{\hat{0}}:=1 .
$$

We say that the labeled poset $P$ is complete if all monomials $m_{F}$ are distinct, and for every $\mathbf{a} \in \mathbb{N}^{n}$ the set $\left\{F \in P: \operatorname{deg}\left(m_{F}\right) \leq \mathbf{a}\right\}$ has a unique maximal element.

We identify the principal ideal $\left\langle m_{F}\right\rangle$ with the free $\mathbb{N}^{n}$-graded $\mathbf{k}[\mathbf{x}]$-module of rank 1 with generator in degree $\operatorname{deg} m_{F}$. If $F, G \in P$ and $F<G$, then $m_{F}$ is a divisor of $m_{G}$. Thus there is an inclusion of the corresponding ideals $i:\left\langle m_{G}\right\rangle \longrightarrow\left\langle m_{F}\right\rangle$. Recall that there is a complex $\mathscr{Z}(P)$ of $\mathbf{k}$-vector spaces associated with $P$. Tensoring summands of this complex with the ideals $\left\{\left\langle m_{F}\right\rangle: F \in P\right\}$, we obtain a complex of 
$\mathbb{N}^{n}$-graded free $\mathbf{k}[\mathbf{x}]$-modules:

$$
\mathscr{C}(P)=\bigoplus_{F \in P} Z_{\mathrm{rk}(F)-2}(\Delta(F)) \otimes_{\mathbf{k}}\left\langle m_{F}\right\rangle \quad \text { with differential } \partial=\phi \otimes i .
$$

\section{THEOREM 3.9}

Suppose that the labeled poset $P$ is complete and that the homology $\widetilde{H}_{i}\left(\Delta\left(F_{(i+3)}\right)\right)$ vanishes for any $0 \leq i \leq r-3$ and any $F \in P$ of rank $\geq i+3$; then $(\mathscr{C}(P), \partial)$ is a minimal $\mathbb{N}^{n}$-graded free $\mathbf{k}[\mathbf{x}]$-resolution of the poset ideal $I_{P}$.

\section{Proof}

$(\mathscr{C}(P), \partial)$ is a complex of $\mathbb{N}^{n}$-graded free $\mathbf{k}[\mathbf{x}]$-modules. To show that it is a resolution, we have to check that, for any $\mathbf{a} \in \mathbb{N}^{n}$, the ath graded component $(\mathscr{C}(P), \partial)_{\mathbf{a}}$ is an exact complex of $\mathbf{k}$-vector spaces. Let $\mathbf{a} \in \mathbb{N}^{n}$, and let $F \in P$ be the maximal element among all elements $G \in P$ such that $\operatorname{deg}\left(m_{G}\right) \leq \mathbf{a}$. Such an element $F$ exists since the labeled poset $P$ is complete. Then $(\mathscr{C}(P), \partial)_{\mathbf{a}}$ is isomorphic to the complex $\mathscr{Z}([\hat{0}, F])$ of the poset $[\hat{0}, F]$ and hence is exact over $\mathbf{k}$ (by Proposition 3.6). Thus $(\mathscr{C}(P), \partial)$ is exact over $\mathbf{k}[\mathbf{x}]$. Finally, since $m_{F}$ and $m_{G}$ are distinct monomials for any pair $F \lessdot G$, the resolution $(\mathscr{C}(P), \partial)$ is minimal.

From Corollary 3.8 we obtain the following corollary.

\section{COROLLARY 3.10}

If $P$ is a complete labeled poset such that every lower interval of $P$ is CohenMacaulay, then $(\mathscr{C}(P), \partial)$ is a minimal $\mathbb{N}^{n}$-graded free resolution of $I_{P}$.

Returning to our matroid $\underline{\mathscr{M}}$, let $P$ be a lattice of flats ordered by reverse inclusion. Hence $P$ is the order dual of the geometric lattice $L$ above. In particular, $\hat{0}$ corresponds to the set $\{1,2, \ldots, n\}$, and $\hat{1}$ corresponds to the empty set. Label each atom $H$ of $P$ (i.e., hyperplane of $\underline{\mathscr{M}}$ ) by the monomial $m_{x}(H)$, as in the beginning of Section 3 . Identifying the variables $x_{i}$ with the coatoms of $P$, we see that $m_{x}(H)$ is the product over all coatoms not above $H$. Then $P$ is a complete labeled poset and its poset ideal $I_{P}$ is precisely the matroid ideal $M$. Moreover, all lower intervals of the poset $P$ are Cohen-Macaulay (see $[16, \S 8]$ ). From Corollary 3.10 we obtain the following alternative to Theorem 3.3.

THEOREM 3.11

Let $\underline{M}$ be any matroid. Then the complex $(\mathscr{C}(P), \partial)$ is a minimal $\mathbb{N}^{n}$-graded free $\mathbf{k}[\mathbf{x}]$-resolution of the matroid ideal $M$. 
The two resolutions presented in this section provide a syzygetic realization of Stanley's formula [16, Th. 9] for the Betti numbers of matroid ideals. That formula states that the number of minimal $i$ th syzygies of $\mathbf{k}[\mathbf{x}] / M$ is equal to

$$
\beta_{i}(M)=\sum_{F}\left|\mu_{L}(F, \hat{1})\right|,
$$

where the sum is over all flats $F$ of corank $i$ in $\underline{M}$. The generating function

$$
\psi_{\underline{\mathscr{M}}}(q)=\sum_{i=0}^{\mathrm{rk}(M)} \beta_{i}(M) \cdot q^{i}=\sum_{F \text { flat of } \underline{\mathscr{M}}}\left|\mu_{L}(F, \hat{1})\right| \cdot q^{\operatorname{corank}(F)}
$$

for the Betti numbers of $M$ is called the cocharacteristic polynomial of $\mathscr{M}$. In the next two sections we examine this polynomial for some special matroids.

\section{Unimodular toric arrangements}

A toric arrangement is a hyperplane arrangement that lives on a torus $\mathbb{T}^{d}$ rather than in $\mathbb{R}^{d}$. One construction of such arrangements appears in recent work of Bayer, Popescu, and Sturmfels [3]. Experts on geometric combinatorics might appreciate the following description: fix a unimodular matroid $\mathscr{M}$, form the associated tiling of Euclidean space by zonotopes (see [21, Prop. 3.3.4]), dualize to get an infinite arrangement of hyperplanes, and divide out by the group of lattice translations.

Here is the same construction again, but now in slow motion. Fix a central hyperplane arrangement $\mathscr{C}=\left\{H_{1}, \ldots, H_{n}\right\}$ in $\mathbb{R}^{d}$, where $H_{i}=\left\{v \in \mathbb{R}^{d}: h_{i} \cdot v=0\right\}$ for some $h_{i} \in \mathbb{Z}^{d}$. Let $L$ denote the intersection lattice of $\mathscr{C}$ ordered by reverse inclusion. We assume that $\mathscr{C}$ is unimodular, which means that the $(d \times n)$-matrix $\left(h_{1}, \ldots, h_{n}\right)$ has rank $d$, and all its $(d \times d)$-minors lie in the set $\{0,1,-1\}$. We retain this hypothesis throughout this section. (See [21] and [3, Th. 1.2] for details on unimodularity.) The set of all integral translates of hyperplanes of $\mathscr{C}$,

$$
H_{i j}=\left\{v \in \mathbb{R}^{d}: h_{i} \cdot v=j\right\} \quad \text { for } i \in\{1, \ldots, n\} \text { and } j \in \mathbb{Z},
$$

forms an infinite arrangement $\widetilde{\mathscr{C}}$ in $\mathbb{R}^{d}$. The unimodularity hypothesis is equivalent to saying that the set of vertices of $\tilde{\mathscr{C}}$ is precisely the lattice $\mathbb{Z}^{d}$; that is, no new vertices can be formed by intersecting the hyperplanes $H_{i j}$. Define the unimodular toric arrangement $\tilde{\mathscr{C}} / \mathbb{Z}^{d}$ to be the set of images of the $H_{i j}$ in the torus $\mathbb{T}^{d}=\mathbb{R}^{d} / \mathbb{Z}^{d}$.

Slightly abusing notation, we refer to these images as hyperplanes on the torus. The images of cells of $\widetilde{\mathscr{C}}$ in $\mathbb{T}^{d}$ are called cells of $\tilde{\mathscr{C}} / \mathbb{Z}^{d}$. These cells form a cellular decomposition of $\mathbb{T}^{d}$. Denote by $f_{i}=f_{i}\left(\widetilde{\mathscr{C}} / \mathbb{Z}^{d}\right)$ the number of $i$-dimensional cells in this decomposition. The next result concerns the $f$-vector $\left(f_{0}, f_{1}, \ldots, f_{d}\right)$ of $\tilde{\mathscr{C}} / \mathbb{Z}^{d}$. 
THEOREM 4.1

If $\widetilde{\mathscr{C}} / \mathbb{Z}^{d}$ is a unimodular toric arrangement, then

$$
\sum_{i=0}^{d} f_{i}\left(\widetilde{\mathscr{C}} / \mathbb{Z}^{d}\right) \cdot q^{i}=\psi_{\mathscr{C}}(q), \quad \text { where } \psi_{\mathscr{C}}(q)=\sum_{F \in L} \mu_{L}(F, \hat{1}) \cdot(-q)^{\operatorname{dim} F}
$$

is the cocharacteristic polynomial of the underlying hyperplane arrangement $\mathscr{C}$.

\section{Proof}

Choose a vector $w \in \mathbb{R}^{d}$ which is not perpendicular to any 1-dimensional cell of the arrangement $\mathscr{C}$. Consider the affine hyperplane $\left\{v \in \mathbb{R}^{d}: w \cdot v=1\right\}$. Let $\mathscr{A}=\mathscr{C} \cap H$ be a restriction of $\mathscr{C}$ to $H$. Then $\mathscr{A}$ is an affine arrangement in $H$. For any $i \geq 0$, there is a one-to-one correspondence between the $(i-1)$-dimensional bounded cells of $\mathscr{A}$ and the $i$-dimensional cells of toric arrangement $\tilde{\mathscr{C}} / \mathbb{Z}^{d}$. To see this, consider the cells in the infinite arrangement $\tilde{\mathscr{C}}$ whose minimum with respect to the linear functional $v \mapsto w \cdot v$ is attained at the origin. These cells form a system of representatives modulo the $\mathbb{Z}^{d}$-action. But they are also in bijection with the bounded cells of $\mathscr{A}$. Using Proposition 3.5 (see also Example 2.1), we conclude

$$
f_{i}\left(\tilde{\mathscr{C}} / \mathbb{Z}^{d}\right)=f_{i-1}\left(B_{\mathscr{A}}\right)=(-1)^{i} \cdot \sum_{\operatorname{dim}(F)=i} \mu_{L}(F, \hat{1}),
$$

where the sum is over elements of $L$ of corank $i$. This completes the proof.

Theorem 4.1 was found independently by V. Reiner, who suggested that we include the following alternative proof. His proof has the advantage that it does not rely on Zaslavsky's formula.

\section{Second proof of Theorem 4.1}

Starting with the unimodular toric arrangement $\tilde{\mathscr{C}} / \mathbb{Z}^{d}$, for each intersection subspace $F$ in the intersection lattice $L$, let $T_{F}$ denote the subtorus obtained by restricting $\tilde{\mathscr{C}} / \mathbb{Z}^{d}$ to $F$. So $T_{0}$ is just $\tilde{\mathscr{C}} / \mathbb{Z}^{d}$ itself, and $T_{1}$ is not actually a torus but rather a point. Our assertion is equivalent to

$$
\mu(F, 1)=(-1)^{\operatorname{dim} F} \cdot \#\left\{\max \text { cells in } T_{F}\right\} .
$$

Let $\mu^{\prime}(F)$ denote the right-hand side above. By the definition of the Möbius function of a poset, equation (9) is equivalent to

$$
\sum_{F \leq G \leq 1} \mu^{\prime}(G)=\delta_{F, 1} \quad \text { (Kronecker delta). }
$$

The left-hand side of this equation is the (nonreduced) Euler characteristic of $T_{F}$. This is zero since $T_{F}$ is a torus, unless $F=1$ so that $T_{F}$ is a point, and then it is 1 . 
We remark that Theorem 4.1 can be generalized to arbitrary toric arrangements $\widetilde{\mathscr{C}} / \mathbb{Z}^{d}$ without the unimodularity hypothesis. The face count formula is a sum of local Möbius function values over all (now more than one) vertices of $\widetilde{\mathscr{C}} / \mathbb{Z}^{d}$. That generalization has interesting applications to hypergeometric functions, and it will be studied in [13]. We know of no natural syzygetic interpretation of the complexes $\widetilde{\mathscr{C}} / \mathbb{Z}^{d}$ when $\mathscr{C}$ is not unimodular. The enumerative applications in Section 5 all involve unimodular arrangements, so we restrict ourselves to this case. We need the following recursion for computing cocharacteristic polynomials.

\section{PROPOSITION 4.2}

Let $H$ be a hyperplane of the arrangement $\mathscr{C}$. Then

$$
\psi_{\mathscr{C}}(q)=\psi_{\mathscr{C} \cap H}(q)+q \cdot \sum_{c} \psi_{\mathscr{C} / c}(q),
$$

where the sum is over all lines $c$ of the arrangement $\mathscr{C}$ that are not contained in $H$.

The lines $c$ of the arrangement $\mathscr{C}$ are the coatoms of the intersection lattice $L$. The arrangement $\mathscr{C} / c$ is the hyperplane arrangement $\left\{H_{i} / c: c \in H_{i}\right\}$ in the $(d-1)$ dimensional vectorspace $\mathbb{R}^{d} / c$. Note that if $c$ is a simple intersection, that is, if $c$ lies on only $d-1$ hyperplanes $H_{i}$, then $\psi_{\mathscr{C} / c}(q)=(1+q)^{d-1}$. Note that Proposition 4.2, together with the condition $\psi_{\mathscr{C}}(q)=1$ for the zero-dimensional arrangement $\mathscr{C}$, uniquely defines the cocharacteristic polynomial.

\section{Proof}

The intersection lattice $L$ of any central hyperplane arrangement $\mathscr{C}$ is semimodular; that is, if both $F$ and $G$ cover $F \wedge G$, then $F \vee G$ covers both $F$ and $G$ (see [18, $\S 3.3 .2])$. The assertion follows from the relation [18, §3.10, (27)] for the Möbius functions of any semimodular lattice.

In the remainder of this section we review the algebraic context in which unimodular toric arrangements arise in [3]. This provides a Gröbner basis interpretation for our proof of Theorem 4.1, and it motivates our enumerative results in Section 5.

Denote by $B$ the $(n \times d)$-matrix whose rows are $h_{1}, \ldots, h_{n}$. All $(d \times d)$-minors of $B$ are $-1,0$, or +1 . The unimodular Lawrence ideal of $\mathscr{C}$ is the binomial prime ideal

$$
J_{\mathscr{C}}:=\left\langle\mathbf{x}^{a} \mathbf{y}^{b}-\mathbf{y}^{a} \mathbf{x}^{b} \mid a, b \in \mathbb{N}^{n}, a-b \in \operatorname{Image}(B)\right\rangle \quad \text { in } \mathbf{k}\left[x_{1}, \ldots, x_{n}, y_{1}, \ldots, y_{n}\right] .
$$

The main result of [3] states that the toric arrangement $\mathscr{C} / \mathbb{Z}^{d}$ supports a cellular resolution of $J_{\mathscr{C}}$. In particular, the Betti numbers of the unimodular Lawrence ideal $J_{\mathscr{C}}$ are precisely the coefficients of the cocharacteristic polynomial $\psi_{\mathscr{C}}(q)$. 
The construction in the proof of Theorem 4.1 has a Gröbner basis interpretation. Indeed, the generic vector $w \in \mathbb{R}^{d}$ defines a term order $\succ$ for the ideal $J_{\mathscr{C}}$ as follows:

$$
\mathbf{x}^{a} \mathbf{y}^{b} \succ \mathbf{y}^{a} \mathbf{x}^{b} \quad \text { if } a-b=B \cdot u \text { for some } u \in \mathbb{R}^{d} \text { with } w \cdot u>0 .
$$

It is shown in $[3, \S 4]$ that the initial monomial ideal $\operatorname{in}_{\succ}\left(J_{\mathscr{C}}\right)$ of $J_{\mathscr{C}}$ with respect to these weights is the oriented matroid ideal associated with the restriction of the central arrangement $\mathscr{C}$ to the affine hyperplane $\left\{v \in \mathbb{R}^{d}: w \cdot v=1\right\}$. In symbols,

$$
\operatorname{in}_{\succ}\left(J_{\mathscr{C}}\right)=O_{\mathscr{A}}
$$

In fact, in the unimodular case, Theorem 1.3(b) is precisely [3, Th. 4.4].

\title{
COROLLARY 4.3
}

The Betti numbers of the unimodular Lawrence ideal $J_{\mathscr{C}}$, and all its initial ideals $\operatorname{in}_{\succ}\left(J_{\mathscr{C}}\right)$, are the coefficients of the cocharacteristic polynomial $\psi_{\mathscr{C}}$.

We close this section with a nontrivial example. Let $n=9, d=4$, and consider

$$
B^{T}=\left(\begin{array}{ccccccccc}
x_{11} & x_{12} & x_{13} & x_{21} & x_{22} & x_{23} & x_{31} & x_{32} & x_{33} \\
1 & -1 & 0 & -1 & 1 & 0 & 0 & 0 & 0 \\
0 & 1 & -1 & 0 & -1 & 1 & 0 & 0 & 0 \\
0 & 0 & 0 & 1 & -1 & 0 & -1 & 1 & 0 \\
0 & 0 & 0 & 0 & 1 & -1 & 0 & -1 & 1
\end{array}\right) .
$$

All nonzero $(4 \times 4)$-minors of this matrix are -1 or +1 , and hence we get a unimodular central arrangement $\mathscr{C}$ of nine hyperplanes in $\mathbb{R}^{4}$. This is the cographic arrangement associated with the complete bipartite graph $K_{3,3}$. The nine hyperplane variables $x_{i j}$ represent edges in $K_{3,3}$. The associated Lawrence ideal can be computed by saturation (e.g., in Macaulay 2) from (binomials representing) the four rows of $B^{T}$ :

$$
J_{B}=\left\langle x_{11} x_{22} y_{12} y_{21}-x_{12} x_{21} y_{11} y_{22}, x_{12} x_{23} y_{13} y_{22}-x_{13} x_{22} y_{12} y_{23},\right.
$$

$\left.x_{21} x_{32} y_{22} y_{31}-x_{22} x_{31} y_{21} y_{32}, x_{22} x_{33} y_{23} y_{32}-x_{23} x_{32} y_{22} y_{33}\right\rangle:\left(\prod_{1 \leq i, j \leq 3} x_{i j} y_{i j}\right)^{\infty}$.

This ideal has 15 minimal generators, corresponding to the 15 circuits in the directed graph $K_{3,3}$. A typical initial monomial ideal $\operatorname{in}_{\prec}\left(J_{B}\right)=O_{\mathscr{A}}$ looks like this:

\author{
$\left\langle x_{11} x_{22} y_{12} y_{21}, x_{11} x_{23} y_{13} y_{21}, x_{11} x_{32} y_{12} y_{31}, x_{11} x_{33} y_{13} y_{31}, x_{12} x_{23} y_{13} y_{22}\right.$, \\ $x_{12} x_{33} y_{13} y_{32}, x_{21} x_{32} y_{22} y_{31}, x_{21} x_{33} y_{23} y_{31}, x_{22} x_{33} y_{23} y_{32}$, \\ $x_{11} x_{22} x_{33} y_{13} y_{21} y_{32}, x_{11} x_{22} x_{33} y_{12} y_{23} y_{31}, x_{11} x_{23} x_{32} y_{13} y_{22} y_{31}$, \\ $\left.x_{12} x_{21} x_{33} y_{11} y_{23} y_{32}, x_{12} x_{21} x_{33} y_{13} y_{22} y_{31}, x_{13} x_{21} x_{32} y_{12} y_{23} y_{31}\right\rangle$.
}


This is the oriented matroid ideal of the 3-dimensional affine arrangement $\mathscr{A}$ gotten from $\mathscr{C}$ by taking a vector $w \in \mathbb{R}^{4}$ with strictly positive coordinates. This ideal is the intersection of 81 monomial primes, one for each spanning tree of $K_{3,3}$. By Theorem 2.9, they form a triangulation of a 13-dimensional Lawrence polytope, which is given by its centrally symmetric Gale diagram $\left(B^{T},-B^{T}\right)$, as in [6, Prop. 9.3.2(b)]. Resolving this ideal (e.g., in Macaulay 2), we obtain the cocharacteristic polynomial

$$
\psi_{\mathscr{C}}(q)=1+15 q+48 q^{2}+54 q^{3}+20 q^{4} .
$$

It was asked in [3, §5] what such Betti numbers arising from graphic and cographic ideals are in general. This question is answered in the following section.

\section{Graphic and cographic matroids}

Among all matroids the unimodular ones play a special role; among unimodular matroids those that arise from graphs play a special role; among all graphs the complete graph plays a special role. Our aim in this section is to compute the cocharacteristic polynomial of graphic and cographic arrangements, with an emphasis on complete graphs. The material in this section is purely combinatorial and can be read independently from the commutative algebra seen earlier. However, the motivation that led us to prove Theorems 5.8 and 5.14 arose from the desire to count minimal syzygies. The results in this section provide answers to questions posed in $[3, \S 4]$. We start out by discussing graphic arrangements. Cographic arrangements are more challenging and are discussed further below.

Fix a connected graph $G$ with vertices $[d]=\{1, \ldots, d\}$ and edges $E \subset[d] \times[d]$. Let $V=\left\{\left(v_{1}, \ldots, v_{d}\right) \in \mathbb{R}^{d}: v_{1}+\cdots+v_{d}=0\right\} \simeq \mathbb{R}^{d-1}$. The graphic arrangement $\mathscr{C}_{G}$ is the arrangement in $V$ given by the hyperplanes $v_{i}=v_{j}$ for $(i, j) \in E$. It is unimodular (see [21]). For each subset $S \subset[d]$, we get an induced subgraph $\left.G\right|_{S}=$ $(S, E \cap(S \times S))$. For a partition $\pi$ of $[d]$, we denote by $G / \pi$ the graph obtained from $G$ by contracting all edges whose vertices lie in the same part of $\pi$. The intersection lattice $L_{G}$ of the graphic arrangement $\mathscr{C}_{G}$ has the following well-known description in terms of the partition lattice $\Pi_{d}$ (see, e.g., [22] for proofs and references).

\section{PROPOSITION 5.1}

The intersection lattice $L_{G}$ is isomorphic to the sublattice of the partition lattice $\Pi_{d}$ consisting of partitions $\pi$ such that, for each part $S$ of $\pi$, the subgraph $\left.G\right|_{S}$ is connected. The element $V_{\pi}$ of $L_{G}$ corresponding to $\pi \in \Pi_{d}$ is the intersection of the hyperplanes $\left\{v_{i}=v_{j}\right\}$ for pairs $i, j$ in the same part of $\pi$. The dimension of $V_{\pi}$ is equal to the number of parts of $\pi$ minus 1 . The interval $\left[V_{\pi}, \hat{1}\right]$ of the intersection lattice $L_{G}$ is isomorphic to the intersection lattice $L_{G / \pi}$.

We write $\mu(G)=\left|\mu_{L_{G}}(\hat{0}, \hat{1})\right|$ for the Möbius invariant of the intersection lattice. 
Thus $\mu(G)$ equals the Cohen-Macaulay type (top Betti number) of the matroid ideal

$$
M_{G}=\bigcap\left\{\left\langle x_{i j}:(i, j) \in F\right\rangle \mid F \subseteq E \text { is a spanning tree of } G\right\} .
$$

From Proposition 5.1 and (8), we conclude that all the lower Betti numbers can be expressed in terms of the Möbius invariants of the contractions $G / \pi$ of $G$.

\section{COROLLARY 5.2}

The cocharacteristic polynomial of the graphic arrangement $\mathscr{C}_{G}$ is

$$
\psi_{\mathscr{C}_{G}}(q)=\sum_{\pi \in L_{G}} \mu(G / \pi) \cdot q^{|\pi|-1} .
$$

This reduces our problem to computing the Möbius invariant $\mu(G)$ of a graph $G$. C. Greene and Zaslavsky [10] found the following combinatorial formula. An orientation of the graph $G$ is a choice, for each edge $(i, j)$ of $G$, of one of the two possible directions: $i \rightarrow j$ or $j \rightarrow i$. An orientation is acyclic if there is no directed cycle.

\section{PROPOSITION 5.3}

Fix a vertex $i$ of $G$. Then $\mu(G)$ equals the number of acyclic orientations of $G$ such that, for any vertex $j$, there is a directed path from $i$ to $j$.

\section{Proof}

The regions of the graphic arrangement $\mathscr{C}_{G}$ are in one-to-one correspondence with the acyclic orientations of $G$ : the region corresponding to an acyclic orientation $o$ is given by the inequalities $x_{i}>x_{j}$ for any directed edge $i \rightarrow j$ in $o$.

The linear functional $w:\left(u_{1}, \ldots, u_{d}\right) \mapsto u_{i}$ is generic for the arrangement $\mathscr{C}_{G}$. The Möbius invariant $\mu(G)$ equals the number of regions of $\mathscr{C}_{G}$ which are bounded below with respect to $w$. We claim that the acyclic orientations corresponding to the $w$-bounded regions are precisely the ones given in our assertion.

Suppose that, for any vertex $j$ in $G$, there is a directed path $i \rightarrow \cdots \rightarrow j$. For any point $\left(u_{1}, \ldots, u_{d}\right)$ of the corresponding region, this path implies $u_{i}>\cdots>u_{j}$. The condition $u_{1}+\cdots+u_{m}=0$ forces $w(u)=u_{i}>0$. This implies that the region is $w$-positive. Conversely, consider any acyclic orientation that does not satisfy the condition in Proposition 5.3. Then there exists a vertex $j \neq i$ which is a source of $o$. Then the vector $v=(-1, \ldots,-1, d-1,-1, \ldots,-1)$, where $d-1$ is in the $j$ th coordinate, belongs to the closure of the region associated with $o$. But $w(v)=-1$. Hence the region is not $w$-positive.

The above discussion can be translated into a combinatorial recipe for writing the minimal free resolution of graphic ideals $M_{G}$, where each syzygy is indexed by a 
certain acyclic orientation of a graph $G / \pi$. For the case of the complete graph $G=$ $K_{d}$, we recover the resolution in [3, Th. 5.3]. Note that the intersection lattice $L_{K_{d}}$ is isomorphic to the partition lattice $\Pi_{d}$. For any partition $\pi$ of $\{1, \ldots, d\}$ with $i+1$ parts, $K_{d} / \pi$ is isomorphic to $K_{i+1}$. The number of such partitions equals $S(d, i+1)$, the Stirling number of the second kind. The number of acyclic orientations of $K_{i+1}$ with a unique fixed source equals $i$ !. We deduce the following corollary.

\section{COROLLARY 5.4}

The number of minimal ith syzygies of $M_{K_{d}}$ equals $i ! S(d, i+1)$.

\section{Remark 5.5}

Reiner suggested to us the following combinatorial interpretation of $\mu(G)$. It can be derived from Proposition 5.3. For any graph $G$, the Möbius invariant $\mu(G)$ counts the number of equivalence classes of linear orderings of the vertices of $G$, under the equivalence relation generated by the following operations:

- $\quad$ commuting two adjacent vertices $v, v^{\prime}$ in the ordering if $\left\{v, v^{\prime}\right\}$ is not an edge of $G$,

- $\quad$ cyclically shifting the entire order, that is, $v_{1} v_{2} \cdots v_{n} \leftrightarrow v_{2} \cdots v_{n} v_{1}$.

Invariance under the second operation makes this interpretation convenient for writing down the minimal free resolution of the graphic Lawrence ideals in $[3, \S 5]$.

Another application arises when $(W, S)$ is a Coxeter system and $G$ its Coxeter graph (considered without its edge labels). Suppose $S=\left\{s_{1}, \ldots, s_{n}\right\}$. Then $\mu(G)$ counts the number of Coxeter elements $s_{i_{1}} \cdots s_{i_{n}}$ of $G$ up to the equivalence relation $s_{i_{1}} s_{i_{2}} \cdots s_{i_{n}} \leftrightarrow s_{i_{2}} \cdots s_{i_{n}} s_{i_{1}}$.

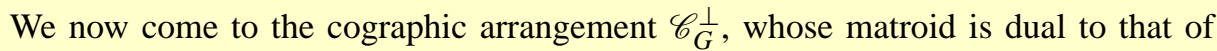
$\mathscr{C}_{G}$. Fix a directed graph $G$ on $[d]$ with edges $E$, where $G$ is allowed to have loops and multiple edges. We associate with $G$ the multiset of vectors $\left\{v_{e} \in \mathbb{Z}^{d}: e \in E\right\}$, where, for an edge $e=(i \rightarrow j)$, the $i$ th coordinate of $v_{e}$ is 1 , the $j$ th coordinate is -1 , and all other coordinates are zero. Set $v_{e}=0$ for a loop $e=(i \rightarrow i)$ of $G$. Let $V_{G}=\left\{\lambda: E \rightarrow \mathbb{R} \mid \sum_{e \in E} \lambda(e) v_{e}=0\right\}$. Note that $V_{G}$ is a vector space of dimension $\#\{$ edges $\}-\#\{$ vertices $\}+\#$ \{connected components $\}$. The cographic arrangement $\mathscr{C}_{G} \frac{1}{G}$ is the arrangement in $V_{G}$ given by hyperplanes $H_{e}=\left\{\lambda \in V_{G}: \lambda(e)=0\right\}$ for $e \in E$. It is unimodular (see [21]). We write $\mu^{\perp}(G)=\left|\mu_{L_{G}^{\perp}}(\hat{0}, \hat{1})\right|$ for the Möbius invariant of the intersection lattice $L_{G}^{\perp}$ of $\mathscr{C}_{G}^{\perp}$, and we refer to this number as the Möbius coinvariant of $G$. Thus $\mu^{\perp}(G)$ is the Cohen-Macaulay type of the cographic ideal $J_{C_{G}^{\perp}}$ in $[3, \S 5]$. 


\section{Remark 5.6}

The characteristic polynomial of a matroid can be expressed via the Tutte dichromatic polynomial (see [20]). Thus the Möbius invariant and coinvariant of a graph $G$ are certain values of the Tutte polynomial: $\mu(G)=T_{G}(1,0)$ and $\mu^{\perp}(G)=T_{G}(0,1)$. We do not know, however, how to express the cocharacteristic polynomial $\psi(q)$ in terms of the Tutte polynomial.

A formula for the Tutte polynomial due to I. Gessel and B. Sagan [9, Th. 2.1] implies the following proposition.

\section{PROPOSITION 5.7}

The Möbius coinvariant of $G$ is $\mu^{\perp}(G)=\sum_{F \subseteq G}(-1)^{d-|F|-1}$, where the sum is over all forests in $G$ and $|F|$ denotes the number of edges in $F$.

We derive explicit formulas for the Möbius coinvariant of complete and complete bipartite graphs. A subgraph $M$ of a graph $G$ is called a partial matching if it is a collection of pairwise disjoint edges of the graph. For a partial matching $M$, let $a(M)$ be the number of vertices of $G$ that have degree zero in $M$. The Hermite polynomial $H_{n}(x), n \geq 0$, is the generating function of partial matchings in the complete graph $K_{n}$ :

$$
H_{n}(x)=\sum_{M} x^{a(M)},
$$

where the sum is over all partial matchings in $K_{n}$. In particular, $H_{0}(x)=1$. Set also $H_{-1}(x)=0$. The main result of this section is the following formula.

\section{THEOREM 5.8}

The Möbius coinvariant of the complete graph $K_{m}$ equals

$$
\mu^{\perp}\left(K_{m}\right)=(m-2) H_{m-3}(m-1), \quad m \geq 2 .
$$

A few initial numbers $\mu^{\perp}\left(K_{m}\right)$ are given below:

$$
\begin{array}{lcccccccccc}
m & 2 & 3 & 4 & 5 & 6 & 7 & 8 & 9 & 10 & \ldots, \\
\mu^{\perp}\left(K_{m}\right) & 0 & 1 & 6 & 51 & 560 & 7575 & 122052 & 2285353 & 48803904 & \ldots
\end{array}
$$

The proof of Theorem 5.8 relies on several auxiliary results and is given below. The next proposition summarizes well-known properties of Hermite polynomials. 
PROPOSITION 5.9

The Hermite polynomial $H_{n}(x)$ satisfies the recurrence

$$
\begin{aligned}
& H_{-1}(x)=0, \quad H_{0}(x)=1, \\
& H_{n+1}(x)=x H_{n}(x)+n H_{n-1}(x), \quad n \geq 0 .
\end{aligned}
$$

It is given explicitly by the formula

$$
H_{n}(x)=x^{n}+\sum_{k \geq 1}^{[n / 2]}\left(\begin{array}{c}
n \\
2 k
\end{array}\right)(2 k-1) ! ! x^{n-2 k},
$$

where $(2 k-1) ! !=(2 k-1)(2 k-3)(2 k-5) \cdots 3 \cdot 1$.

\section{Proof}

In a partial matching the first vertex has either degree 0 or 1 . This gives two terms in the right-hand side of the recurrence (12). The formula for $H_{n}(x)$ follows from the fact that there are $(2 k-1)$ !! matchings with $k$ edges on $2 k$ vertices.

Returning to general cographic arrangements, recall that an edge $e$ of the graph $G$ is called an isthmus if $G \backslash e$ has more connected components than $G$; a graph is called isthmus-free if no edge of $G$ is an isthmus. The minimal nonempty isthmus-free subgraphs of $G$ are the cycles of $G$. For a subgraph $H$ of $G$, denote by $G / H$ the graph obtained from $G$ by contracting the edges of $H$. Note that $G / H$ may have loops and multiple edges even if $G$ does not. The following result appears in [10].

\section{PROPOSITION 5.10}

The intersection lattice $L_{G}^{\perp}$ of the cographic arrangement is isomorphic to the lattice of isthmus-free subgraphs of $G$ ordered by reverse inclusion. The element of the intersection lattice that corresponds to an isthmus-free subgraph $H$ is $V_{H} \subset V_{G}$. The coatoms of the lattice $L_{G}^{\perp}$ are the cycles of $G$. For two isthmus-free subgraphs $H \supset K$ of $G$, the interval $\left[V_{H}, V_{K}\right]$ of the intersection lattice $L_{G}^{\perp}$ is isomorphic to the interval $[\hat{0}, \hat{1}]$ of the intersection lattice $L_{H / K}^{\perp}$.

Proposition 4.2 implies the following recurrence for the cocharacteristic polynomial $\psi_{\mathscr{C}_{G}^{\perp}}(q)$ of the cographic arrangement $\mathscr{C}_{G}^{\perp}$.

\section{COROLLARY 5.11}

Let $e$ be an edge of the graph $G$. Then

$$
\psi_{\mathscr{C}_{G}^{\perp}}(q)=\psi_{\mathscr{C}_{G \backslash e}^{\perp}}(q)+q \sum_{C} \psi_{\mathscr{C}_{G / C}^{\perp}}(q),
$$

where the sum is over all cycles $C$ of $G$ that contain $e$. 
Considering terms of the highest degree in (13), we obtain the following corollary.

\section{COROLLARY 5.12}

If $e$ is any edge of $G$ that is not an isthmus, then

$$
\mu^{\perp}(G)=\sum_{C} \mu^{\perp}(G / C)
$$

where the sum is over all cycles $C$ of $G$ that contain $e$.

Note that $\mu^{\perp}(G)$ is equal to the Möbius coinvariant of the graph $\widetilde{G}$ obtained from $G$ by removing all loops and isthmuses. Thus, when we use relation (14) to calculate $\mu^{\perp}(G)$, we may remove all new loops obtained after contracting the cycle $C$.

We are ready to prove Theorem 5.8. For $n \geq 0$ and $k \geq 1$, define $K_{n}^{(k)}$ to be the complete graph $K_{n}$ on the vertices $1, \ldots, n$, together with one additional vertex $n+1$ (root) connected to each vertex $1, \ldots, n$ by $k$ edges. Let $\mu_{n}^{(k)}=\mu^{\perp}\left(K_{n}^{(k)}\right)$ be the Möbius coinvariant of the graph $K_{n}^{(k)}$. Note that $K_{m}=K_{m-1}^{(1)}$ and $\mu^{\perp}\left(K_{m}\right)=\mu_{m-1}^{(1)}$. Theorem 5.8 can be extended as follows.

PROPOSITION 5.13

We have the following formula: $\mu_{n}^{(k)}=H_{n}(n+k-1)-n H_{n-1}(n+k-1)$ for $n, k \geq 1$.

\section{Proof}

We utilize Corollary 5.12. Select an edge $e=(n, n+1)$ of the graph $K_{n}^{(k)}$. There are $k-1$ choices for a cycle $C$ of length 2 that contains the edge $e$, and the graph $K_{n}^{(k)} / C$, after removing loops, is isomorphic to $K_{n-1}^{(k+1)}$. There are $(n-1) k$ choices for a cycle $C$ of length 3 that contains the edge $e$, and the graph $K_{n}^{(k)} / C$, after removing loops, is isomorphic to $K_{n-2}^{(k+2)}$. In general, for cycles of length $l \geq 3$, there are $k(n-$ 1) $(n-2) \cdots(n-l+2)$ choices, and we obtain a graph that is isomorphic to $K_{n-l+1}^{(k+l-1)}$. Equation (14) implies the following recurrence for $\mu_{n}^{(k)}$ :

$$
\begin{aligned}
\mu_{n}^{(k)}= & (k-1) \mu_{n-1}^{(k+1)}+k(n-1) \mu_{n-2}^{(k+2)} \\
& +k(n-1)(n-2) \mu_{n-3}^{(k+3)}+k(n-1)(n-2)(n-3) \mu_{n-4}^{(k+4)}+\cdots,
\end{aligned}
$$

which, together with the initial condition $\mu_{0}^{(k)}=1$, defines the numbers $\mu_{n}^{(k)}$ uniquely. Set

$$
b_{n}^{(k)}=\mu_{n}^{(k)}+n \mu_{n-1}^{(k+1)}+n(n-1) \mu_{n-2}^{(k+2)}+\cdots+n(n-1) \cdots 1 \mu_{0}^{(k+n)} .
$$

Then $\mu_{n}^{(k)}=b_{n}^{(k)}-n b_{n-1}^{(k+1)}$ and the relation (15) can be rewritten as

$$
b_{n}^{(k)}-n b_{n-1}^{(k+1)}=(k-1)\left(b_{n-1}^{(k+1)}-(n-1) b_{n-2}^{(k+2)}\right)+k(n-1) b_{n-2}^{(k+2)}
$$


or, simplifying, as

$$
b_{n}^{(k)}=(n+k-1) b_{n-1}^{(k+1)}+(n-1) b_{n-2}^{(k+2)} .
$$

We claim that $b_{n}^{(k)}=H_{n}(n+k-1)$. Indeed, $b_{0}^{(k)}=1, b_{1}(k)=k$, and equation (16) is equivalent to the defining relation (12) for the Hermite polynomials. Hence $\mu_{n}^{(k)}=$ $b_{n}^{(k)}-n b_{n-1}^{(k+1)}=H_{n}(n+k-1)-n H_{n-1}(n+k-1)$.

\section{Proof of Theorem 5.8}

By Proposition 5.13 and equation (12),

$$
\mu^{\perp}\left(K_{m}\right)=\mu_{m-1}^{(1)}=H_{m-1}(m-1)-(m-1) H_{m-2}(m-1)=(m-2) H_{m-3}(m-1) .
$$

We now discuss a bipartite analog of Hermite polynomials. For a partial matching $M$ in the complete bipartite graph $K_{m, n}$, denote by $a(M)$ the number of vertices in the first part that have degree zero in $M$, and by $b(M)$ the number of vertices in the second part that have degree zero. Define

$$
H_{m, n}(x, y)=\sum_{M} x^{a(M)} y^{b(M)},
$$

where the sum is over all partial matchings in $K_{m, n}$. In particular, $H_{m, 0}=x^{m}$ and $H_{0, n}=y^{n}$. Set also $H_{m,-1}=H_{-1, n}=0$. The following statement is a bipartite analogue of Theorem 5.8.

\section{THEOREM 5.14}

The Möbius coinvariant of the complete bipartite graph $K_{m, n}$ equals

$$
\mu^{\perp}\left(K_{m, n}\right)=(m-1)(n-1) H_{m-2, n-2}(n-1, m-1), \quad m, n \geq 1 .
$$

The following proposition is analogous to Proposition 5.9.

PROPOSITION 5.15

The polynomial $H_{m, n}(x, y)$ is given by

$$
H_{m, n}(x, y)=\sum_{k=0}^{\min (m, n)}\left(\begin{array}{l}
m \\
k
\end{array}\right)\left(\begin{array}{l}
n \\
k
\end{array}\right) k ! x^{m-k} y^{n-k} .
$$

It satisfies the following recurrence relations:

$$
\begin{aligned}
H_{m, n}(x, y) & =x H_{m-1, n}(x, y)+n H_{m-1, n-1}(x, y), \\
H_{m, n}(x, y) & =y H_{m, n-1}(x, y)+m H_{m-1, n-1}(x, y), \\
H_{m, 0} & =x^{m}, \quad H_{0, n}=y^{n} .
\end{aligned}
$$




\section{Proof}

The first formula is obtained by counting the partial matchings in $K_{m, n}$. The recurrence relations (17) are obtained by distinguishing two cases when the first vertex in the first (second) part of $K_{m, n}$ has degree 0 or 1 in a partial matching.

Let us define the graph $K_{m, n}^{(k, l)}$ as the complete bipartite graph $K_{m, n}$ with an additional vertex $v$ such that $v$ is connected by $k$ edges with each vertex in the first part and by $l$ edges with each vertex in the second part. Let $\mu_{m, n}^{(k, l)}=\mu^{\perp}\left(K_{m, n}^{(k, l)}\right)$ be the Möbius coinvariant of this graph. Note that $K_{m, n}=K_{m, n-1}^{(1,0)}$ and, thus, $\mu^{\perp}\left(K_{m, n}\right)=\mu_{m, n-1}^{(1,0)}$. Theorem 5.14 can be extended as follows.

\section{PROPOSITION 5.16}

We have

$$
\mu_{m, n}^{(k, l)}=H_{m, n}(n+k-1, m+l-1)-m n H_{m-1, n-1}(n+k-1, m+l-1) .
$$

\section{Proof}

Our proof is similar to that of Proposition 5.13. We utilize Corollary 5.12. Select an edge $e$ of the graph $K_{m, n}^{(k, l)}$ that joins the additional vertex $v$ with a vertex from the first part. There are $k-1$ choices for a cycle $C$ of length 2 that contains the edge $e$, and the graph $K_{m, n}^{(k, l)} / C$, after removing loops, is isomorphic to $K_{m-1, n}^{(k, l+1)}$. There are $n l$ choices for a cycle $C$ of length 3 that contains the edge $e$, and the graph $K_{m, n}^{(k, l)} / C$, after removing loops, is isomorphic to $K_{m-1, n-1}^{(k+1, l+1)}$. For cycles of length 4 , we have $n(m-1) k$ choices and obtain a graph isomorphic to $K_{m-2, n-1}^{(k+1, l+2)}$, and so on. In general, for cycles of odd length $2 r+1 \geq 3$, we have $\ln (m-1)(n-1)(m-2) \cdots(m-r+$ 1) $(n-r+1)$ choices, and we obtain a graph isomorphic to $K_{m-r, n-r}^{(k+r, l+r)}$. For cycles of even length $2 r+2 \geq 4$, we have $k n(m-1)(n-1)(m-2) \cdots(n-r+1)(m-r)$ choices, and we obtain a graph isomorphic to $K_{m-r-1, n-r}^{(k+r, l+r+1)}$. Equation (14) implies the following recurrence for $\mu_{m, n}^{(k, l)}$ :

$$
\begin{aligned}
\mu_{m, n}^{(k, l)}= & (k-1) \mu_{m-1, n}^{(k, l+1)}+\ln \mu_{m-1, n-1}^{(k+1, l+1)}+k n(m-1) \mu_{m-2, n-1}^{(k+1, l+2)} \\
& +\ln (m-1)(n-1) \mu_{m-2, n-2}^{(k+2, l+2)} \\
& +k n(m-1)(n-1)(m-2) \mu_{m-3, n-2}^{(k+2, l+3)}+\cdots,
\end{aligned}
$$

which, together with the initial conditions $\mu_{0, n}^{(k, l)}=(l-1)^{n}$ and $\mu_{m, 0}^{(k, l)}=(k-1)^{m}$, unambiguously defines the numbers $\mu_{m, n}^{(k, l)}$. Let us fix the numbers $p=k+n-1$ and $q=l+m-1$ and write $\mu_{m, n}$ for $\mu_{m, n}^{(p-n+1, q-m+1)}$. Set

$$
b_{m, n}=\mu_{m, n}+n m \mu_{m-1, n-1}+n(n-1) m(m-1) \mu_{m-2, n-2}+\cdots .
$$


Then $\mu_{m, n}=b_{m, n}-m n b_{m-1, n-1}$ and the relation (18) can be rewritten as

$$
\begin{aligned}
b_{m, n}-m n b_{m-1, n-1}= & -\left(b_{m-1, n}-(m-1) n b_{m-2, n-1}\right) \\
& +(p-n+1) b_{m-1, n}+(q-m+1) n b_{m-1, n-1}
\end{aligned}
$$

or, simplifying, as

$$
b_{m, n}=(p-n) b_{m-1, n}+(q+1) n b_{m-1, n-1}+(m-1) n b_{m-2, n-1} .
$$

This relation, together with the initial conditions $b_{0, n}=q^{n}, b_{m, 0}=p^{m}, b_{-1, n}=$ $b_{m,-1}=0$, uniquely determines the numbers $b_{m, n}$.

We claim that $b_{m, n}=H_{m, n}(p, q)$. Indeed, the above initial conditions are satisfied by $H_{m, n}(p, q)$, and (19) follows from the defining relations (17) for the bipartite Hermite polynomials. In order to see this, we write by (17),

$$
\begin{aligned}
H_{m, n}(p, q) & =p H_{m-1, n}(p, q)+n H_{m-1, n-1}(p, q), \\
n H_{m-1, n}(p, q) & =n q H_{m-1, n-1}(p, q)+n(m-1) H_{m-2, n-1}(p, q) .
\end{aligned}
$$

The sum of these two equations is equivalent to equation (19). Hence $\mu_{m, n}^{(k l)}=b_{m, n}-$ $m n b_{m-1, n-1}=H_{m, n}(p, q)-m n H_{m-1, n-1}(p, q)$.

An alternative expression for $\mu_{m, n}^{(k l)}$ can be deduced from Proposition 5.16:

$$
\mu_{m, n}^{(k l)}=\sum_{r=0}^{\min (m, n)}(1-r)\left(\begin{array}{l}
m \\
r
\end{array}\right)\left(\begin{array}{l}
n \\
r
\end{array}\right) r !(n+k-1)^{m-r}(m+l-1)^{n-r} .
$$

\section{Proof of Theorem 5.14}

By Proposition 5.16 and the recurrence relations (17),

$$
\begin{aligned}
\mu^{\perp} & \left(K_{m, n}\right) \\
& =\mu_{m, n-1}^{(1,0)}=H_{m, n-1}(n-1, m-1)-m(n-1) H_{m-1, n-2}(n-1, m-1) \\
& =(n-1) H_{m-1, n-1}(n-1, m-1)-(m-1)(n-1) H_{m-1, n-2}(n-1, m-1) \\
& =(m-1)(n-1) H_{m-2, n-2}(n-1, m-1) .
\end{aligned}
$$

For a commutative algebra example illustrating Theorem 5.14, consider the Lawrence ideal $J_{B} \subset \mathbf{k}\left[x_{11}, \ldots, x_{33}, y_{11}, \ldots, y_{33}\right]$ associated with the bipartite graph $K_{3,3}$. This is the Lawrence lifting of the ideal of $(2 \times 2)$-minors of a generic $(3 \times 3)$-matrix. It is discussed in the end of Section 4. Its Cohen-Macaulay type is

$$
\mu^{\perp}\left(K_{3,3}\right)=(3-1) \cdot(3-1) \cdot H_{1,1}(2,2)=2 \cdot 2 \cdot 5=20 .
$$

This is the leading coefficient of the cocharacteristic polynomial in equation (10). 
Acknowledgments. We wish to thank Vic Reiner and Günter Ziegler for valuable communications. Their ideas and suggestions have been incorporated in Proposition 2.4, Theorem 4.1, and Remark 5.5.

\section{References}

[1] E. BATZIES and V. WELKER, Discrete Morse theory for cellular resolutions, preprint,

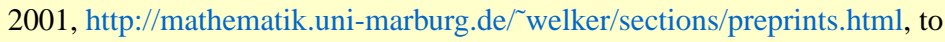
appear in J. Reine Angew. Math. 288

[2] D. BAYER, I. PEEVA, and B. STURMFELS, Monomial resolutions, Math. Res. Lett. 5 (1998), 31-46. MR 99c:13029 288

[3] D. BAYER, S. POPESCU, and B. STURMFELS, Syzygies of unimodular Lawrence ideals, J. Reine Angew. Math. 534 (2001), 169-186. MR CMP 1831636 287, 288, 291, 293, 303, 305, 306, 307, 309

[4] D. BAYER and B. STURMFELS, Cellular resolutions of monomial modules, J. Reine Angew. Math. 502 (1998), 123-140. MR 99g:13018 287, 288

[5] A. BJÖRNER, "The homology and shellability of matroids and geometric lattices" in Matroid Applications, ed. N. White, Encyclopedia Math. Appl. 40, Cambridge Univ. Press, Cambridge, 1992, 226-283. MR 94a:52030 299

A. BJÖRNER, M. LAS VERGNAS, B. STURMFELS, N. WHITE, and G. ZIEGLER, Oriented Matroids, 2 d ed., Encyclopedia Math. Appl. 46, Cambridge Univ. Press, Cambridge, 1999. MR 2000j:52016 290, 291, 292, 293, 294, 295, 298, 299, 307

D. BUCHSBAUM and D. EISENBUD, What makes a complex exact?, J. Algebra 25 (1973), 259-268. MR 47:3369 291

V. GASHAROV, I. PEEVA, and V. WELKER, The lcm-lattice in monomial resolutions, Math. Res. Lett. 6 (1999), 521-532. MR 2001e:13018 298

I. M. GESSEL and B. E. SAGAN, The Tutte polynomial of a graph, depth-first search, and simplicial complex partitions, Electron. J. Combin. 3, no. 2 (1996), research paper 9, http://combinatorics.org/Volume_3/volume3_2.html\#R9 MR 97d:05149 310

[10] C. GREENE and T. ZASLAVSKY, On the interpretation of Whitney numbers through arrangements of hyperplanes, zonotopes, non-Radon partitions, and orientations of graphs, Trans. Amer. Math. Soc. 280 (1983), 97-126. MR 84k:05032 308, 311

E. MILLER and B. STURMFELS, "Monomial ideals and planar graphs" in Applied Algebra, Algebraic Algorithms and Error-Correcting Codes (Honolulu, 1999), ed. M. Fossorier, H. Imai, S. Lin, and A. Poli, Lecture Notes in Comput. Sci. 1719, Springer, Berlin, 1999, 19-28. MR CMP 1846480288

J. R. MUNKRES, Elements of Algebraic Topology, Addison-Wesley, Menlo Park, Calif., 1984. MR 85m:55001 287

A. POSTNIKOV and B. STURMFELS, Toric arrangements and hypergeometric functions, in preparation. 305

V. REINER and V. WELKER, Linear syzygies of Stanley-Reisner ideals, Math. Scand. 89 (2001), 117-132. MR CMP 1856984297 
[15] F. SANTOS, Triangulations of oriented matroids, to appear in Mem. Amer. Math. Soc. 296, 297

[16] R. P. STANLEY, "Cohen-Macaulay complexes" in Higher Combinatorics (Berlin, 1976), ed. M. Aigner, NATO Adv. Study Inst. Ser., Ser. C: Math. and Phys. Sci. 31, Reidel, Dordrecht, 1977, 51-62. MR 58:28010 291, 297, 298, 302, 303

[17] - Balanced Cohen-Macaulay complexes, Trans. Amer. Math. Soc. 249 (1979), 139-157. MR 81c:05012 301

[18] - Enumerative Combinatorics, I, Wadsworth Brooks/Cole Math. Ser., Wadsworth \& Brooks/Cole Adv. Books Software, Monterey, Calif., 1986. MR 87j:05003 305

[19] - Combinatorics and Commutative Algebra, 2d ed., Progr. Math. 41, Birkhäuser, Boston, 1996. MR 98h:05001 296

[20] W. T. TUTTE, On dichromatic polynomials, J. Combin. Theory 2 (1967), 301-320. MR 36:6320 310

[21] N. WHITE, "Unimodular matroids" in Combinatorial Geometries, ed. N. White, Encyclopedia Math. Appl. 29, Cambridge Univ. Press, Cambridge, 1987, 40-52. MR CMP 921067 303, 307, 309

[22] T. ZASLAVSKY, Facing up to arrangements: Face-count formulas for partitions of space by hyperplanes, Mem. Amer. Math. Soc. 1 (1975), no. 154. MR 50:9603 299, 307

Novik

Department of Mathematics, University of Washington, Seattle, Washington 98125-3810, USA; novik@math.washington.edu

\section{Postnikov}

Department of Mathematics, Massachusetts Institute of Technology, Cambridge, Massachusetts 02139-4307, USA; apost@math.mit.edu

\section{Sturmfels}

Department of Mathematics, University of California, Berkeley, California 94720-3840, USA; bernd@math.berkeley.edu 